\title{
Efficient generation of extreme terahertz harmonics in three-dimensional Dirac semimetals
}

\author{
Jeremy Lim $\odot,{ }^{1}$ Yee Sin Ang $\odot,{ }^{1, *}$ F. Javier García de Abajo $\odot,{ }^{2,3}$ Ido Kaminer $\odot,{ }^{4}$ Lay Kee Ang $\odot,{ }^{1, \dagger}$ and Liang Jie Wong $\oplus^{5, *}$ \\ ${ }^{1}$ Science, Math, and Technology, Singapore University of Technology and Design, 8 Somapah Road, Singapore, Singapore 487372 \\ ${ }^{2}$ ICFO-Institut de Ciencies Fotoniques, Barcelona Institute of Science and Technology, 08860 Castelldefels (Barcelona), Spain \\ ${ }^{3}$ ICREA-Institució Catalana de Recerca i Estudis Avançats, Passeig Lluís Companys 23, 08010 Barcelona, Spain \\ ${ }^{4}$ Department of Electrical Engineering, Technion, Haifa 3200003, Israel \\ ${ }^{5}$ School of Electrical and Electronic Engineering, Nanyang Technological University, 50 Nanyang Avenue, Singapore, Singapore 639798
}

(Received 15 June 2020; revised 19 August 2020; accepted 14 October 2020; published 18 November 2020)

\begin{abstract}
We show that three-dimensional (3D) Dirac semimetals (DSMs) can achieve highly efficient terahertz highorder harmonic generation (HHG) up to the 31 st harmonic with input intensities $\approx 10 \mathrm{MW} / \mathrm{cm}^{2}$-over $10^{5}$ times lower than required in conventional terahertz HHG systems. Our theory reveals that this extreme nonlinearity is made possible by the existence of an operation regime that differs from previous demonstrations of lower order harmonic generation. We also reveal an unexpected regime in which emitted harmonics abruptly become negligible beyond the third order. This unprecedented vanishing of higher order nonlinearity has a geometrical origin related to the combination of conical dispersion and extra dimensionality in 3D DSMs, breaking the common notion that 3D DSMs share the essential physics of two-dimensional DSMs. Our findings pave the way to unlocking the full potential of 3D DSMs as efficient platforms for terahertz light sources and optoelectronics at moderate intensities.
\end{abstract}

DOI: 10.1103/PhysRevResearch.2.043252

\section{INTRODUCTION}

The ability to tailor nonlinear optical processes through the use of, for example, conventional nonlinear bulk media [1,2], photonic crystals [3-5], metamaterials [6-8], and quantum materials [9-12] is crucial to the development of photonic technologies and the study of natural phenomena. High-order harmonic generation (HHG) is a nonlinear process involving light emission at integer multiples of the driving laser frequency [9-30]. However, HHG typically requires strong driving fields exceeding $1 \mathrm{GV} / \mathrm{m}\left(\approx \mathrm{TW} / \mathrm{cm}^{2}\right.$ intensities $)$ [17-26]. With rapidly growing interest in the terahertz $(\mathrm{THz})$ regime as the answer to next-generation computation, imaging, and communications technologies, efficient solid-state THz HHG using modest input intensities is a highly soughtafter goal.

Here, we show that three-dimensional (3D) Dirac semimetals (DSMs) are promising solid-state platforms for highly efficient generation of extreme, intraband-dominated $\mathrm{THz}$ harmonics up to the 31 st order and beyond using input field intensities $\approx 10 \mathrm{MW} / \mathrm{cm}^{2}$-an intensity reduction of $>10^{5}$ times compared to previous THz HHG demonstrations.

\footnotetext{
*yeesin_ang@sutd.edu.sg

†ricky_ang@sutd.edu.sg

*liangjie.wong@ntu.edu.sg
}

Published by the American Physical Society under the terms of the Creative Commons Attribution 4.0 International license. Further distribution of this work must maintain attribution to the author(s) and the published article's title, journal citation, and DOI.
In particular, by driving a $\mathrm{Cd}_{3} \mathrm{As}_{2}$ thin film of $250 \mathrm{~nm}$ thickness with peak input fields $\lesssim 10 \mathrm{MV} / \mathrm{m}$, we show that the 31 st harmonic can be brought within five orders of magnitude of the driving harmonic with energy conversion efficiencies exceeding $10^{-5}$. In contrast, conventional solid-state $\mathrm{THz}$ HHG requires driving fields approaching $10 \mathrm{GV} / \mathrm{m}$-in both theoretical prediction and experimental demonstration-just to bring the 22nd harmonic within eight orders of magnitude of the driving harmonic [18]. Crucially, our closed-form analytical expressions for the nonperturbative optical response of 3D DSMs reveal that the combination of experimentally feasible parameters that we have chosen is within a unique operation regime in which highly efficient $\mathrm{THz}$ HHG up to the 31 st order and beyond is possible. We also show that this distinct and unexplored regime of extreme nonlinearity is highly complementary to recent demonstrations showing efficient generation of up to the third [31] and seventh [32] harmonics in 3D DSMs.

Our work also reveals a regime where higher order optical nonlinearities in 3D DSMs completely vanish. In this second regime, the efficiency of HHG beyond the third harmonic is greatly suppressed. We show that the existence of this regime in 3D DSMs has a geometric origin related to their extra dimensionality compared to two-dimensional (2D) DSMs, which have no equivalent regime of suppression. This breaks the common notion that 3D DSMs share the same essential physics as 2D DSMs, which are known for their strong nonlinearity [9-12,33-44]. To quantify this effect, we identify a new physical quantity, the critical potential, that divides the regime of vanishing higher order nonlinearity from the regime where higher order nonlinearities emerge. This quantity also determines the degree of optical nonlinearity exhibited in the latter regime. 
(a)

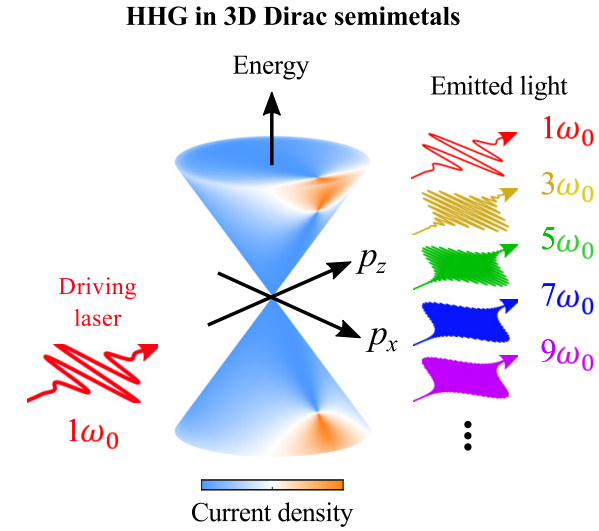

(b) $\mathrm{Cd}_{3} \mathrm{As}_{2}$ thin film driven by a $1 \mathrm{THz}$ pulse (10 MV/m)
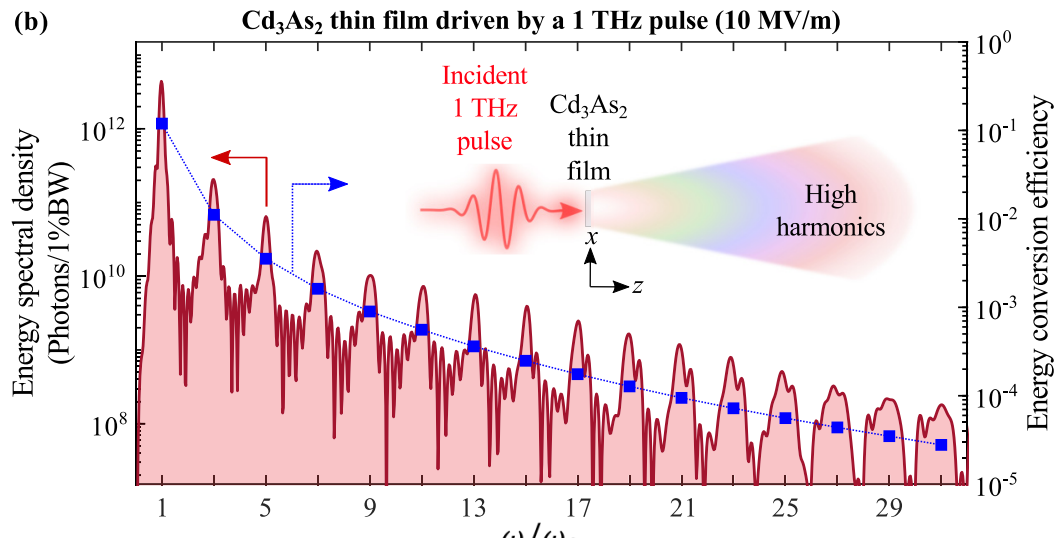
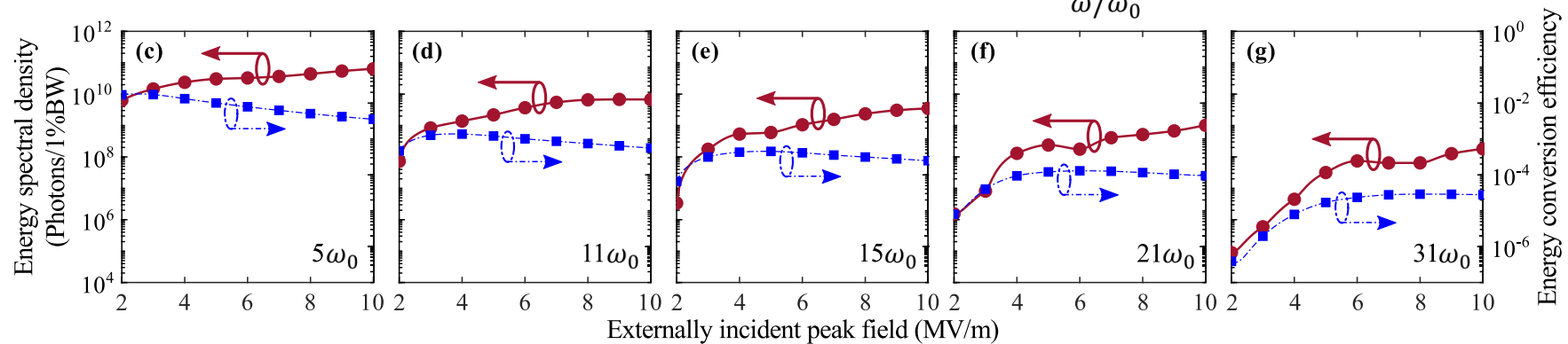

FIG. 1. Highly efficient generation of extreme harmonics (up to 31st order) in the 3D DSM material $\mathrm{Cd}_{3} \mathrm{As}_{2}$ at modest driving field strengths. (a) HHG in a 3D DSM occurs when a driving laser pulse (incoming red wave) induces carrier oscillations (current density depicted at one instant in time on the Dirac cone) and transitions that lead to the emission of high-harmonic light (outgoing multicolored waves). Driving a $\mathrm{Cd}_{3} \mathrm{As}_{2}$ thin film with a linearly polarized pulse of central frequency $1 \mathrm{THz}$ and peak field strength of $10 \mathrm{MV} / \mathrm{m}[(\mathrm{b})$ inset $]$ produces the emitted spectrum shown in panel (b), where we see that harmonics up to the 31 st order and beyond can be generated at energy conversion efficiencies well beyond $10^{-5}$. Panels $[(\mathrm{c})-(\mathrm{g})]$ show the change in the output energy spectrum and conversion efficiency as a function of field strength of the external driving laser (markers, with connecting lines as visual guides). We see that extreme harmonic generation continues to remain relatively efficient even at much lower field strengths. We consider a 250 -nm-thick $\mathrm{Cd}_{3} \mathrm{As}_{2}$ thin film of radius $1 \mathrm{~mm}$ and Fermi energy $\mathcal{E}_{\mathrm{F}}=60 \mathrm{meV}$, uniformly illuminated by a 2-ps long pulse of $1-\mathrm{THz}$ peak frequency.

\section{MODELING NONPERTURBATIVE ELECTRON DYNAMICS IN DSMs}

HHG in DSMs occurs when an impinging laser pulse induces carrier oscillations within (intraband current) and carrier transitions between (interband current) the upper and lower bands of the Dirac cone band structure, resulting in light emission at multiples of the driving laser frequency [Fig. 1(a); overall setup shown in Fig. 1(b) inset]. We model the interaction of the driving laser pulse with the DSM using nonpertubative time-domain quantum simulations for the optical response of the DSM, which then determines the electrodynamics via Maxwell's equations. The key points of our theoretical treatment are summarized as follows (full details in Appendix A).

To model the carrier dynamics in DSMs, we use the timedependent Dirac equation (TDDE) $\mathrm{i} \hbar \partial_{t}=\sum_{j} v_{j} \sigma_{j} p_{j}$, where $t$ is time, $\hbar$ is the reduced Planck constant, $v_{j}$ are the Fermi velocities along Cartesian directions $j, \sigma_{j}$ are the Pauli matrices, and $p_{j}$ are the initial electron momenta, with $j \in\{x, y, z\}$ and $j \in\{x, y\}$ for 3D and 2D DSMs, respectively. We include the driving laser via a modified minimal coupling substitution $\mathbf{p} \rightarrow \pi(t)=\mathbf{p}+e \mathbf{a}(t)$, where $e$ is the elementary charge and $\mathbf{a}(t)$ is the modified vector potential inside the DSM, which is related to the electric field $\mathbf{E}(t)$ by the expression $\mathbf{a}(t)=$ $-e^{-t / \tau} \int_{-\infty}^{t} \mathbf{E}\left(t^{\prime}\right) e^{t^{\prime} / \tau} d t^{\prime} \quad[44]$, with $\tau$ being the inelastic intraband scattering time. In the absence of intraband scattering (i.e., $\tau \rightarrow \infty), \mathbf{a}(t)$ is exactly the vector potential $\mathbf{A}(t)$. From the TDDE, we obtain the total induced current $J_{i}(t)$ in terms of the population inversion $\mathcal{N}_{\mathbf{p}}(t)$ and the interband coherence $\Gamma_{\mathbf{p}}(t)$ (see definitions in Appendix A 1) as

$$
\begin{aligned}
J_{i}= & \frac{g e v_{i}}{(2 \pi \hbar)^{n}} \int\left\{\left(\mathcal{N}_{\mathbf{p}}+1\right) \frac{v_{i} \pi_{i}}{\mathcal{E}}-2 \Lambda_{i} \operatorname{Re}\left(\Gamma_{\mathbf{p}}\right)\right. \\
& \left.+2 \Delta_{i} \operatorname{Im}\left(\Gamma_{\mathbf{p}}\right)\right\} d^{n} \mathbf{p},
\end{aligned}
$$

where $g=4$ is the combined valley and spin degeneracy, and the integral extends over all momentum space. For 3D DSMs, $n=3$ and $i \in\{x, y, z\}$; for 2D DSMs, $n=2$ and $i \in\{x, y\}$. We also define the instantaneous energy $\mathcal{E}(t)=\sqrt{\sum_{i} v_{i}^{2} \pi_{i}^{2}(t)}$, $\left(\Lambda_{x}, \Lambda_{y}, \Lambda_{z}\right)=(\cos \theta \cos \phi, \cos \theta \sin \phi,-\sin \theta)$, and $\left(\Delta_{x}\right.$, $\left.\Delta_{y}, \Delta_{z}\right)=(-\sin \phi, \cos \phi, 0)$, where $\theta(t)=\arccos \left[v_{z} \pi_{z}(t) /\right.$ $\mathcal{E}(t)]$ and $\phi(t)=\arctan \left[v_{y} \pi_{y}(t) / v_{x} \pi_{x}(t)\right]$. For 2D DSMs, we set $\theta=\pi / 2$ and $J_{z}=v_{z}=0$. When we further set $v_{x}=v_{y}=$ $v_{\mathrm{F}}$, we obtain an expression that reduces to the special case of isotropic 2D DSMs in Refs. [10,41-44]. In Eq. (1), the first term of the integrand represents the contribution of the intraband current. The other terms represent the contribution of the interband current. The effect of finite temperature 
and interband carrier scattering is taken into account through $\mathcal{N}_{\mathbf{p}}(t)$ and $\Gamma_{\mathbf{p}}(t)$.

We identify a physical quantity, the critical potential $\Phi_{\text {crit }} \equiv \mathcal{E}_{\mathrm{F}} / e$, which separates the regime of extreme nonlinearity from the regime of vanishing higher order nonlinearity in 3D DSMs. Physically, $\Phi_{\text {crit }}$ represents the minimum momentum space displacement needed by the driving vector potential in order to drive an electron from the Fermi surface to the Dirac point. To proceed, we define the potential $\Phi(t) \equiv$ $\sqrt{\sum_{i} v_{i}^{2} A_{i}^{2}(t)}$. When $\Phi(t)<\Phi_{\text {crit }}$, we obtain the current in the Cartesian direction $i$ from Eq. (1) as

$$
J_{i}^{3 \mathrm{D}, \mathrm{sub}}(t)=-\frac{g e^{2} v_{i}}{6 \pi^{2} \hbar^{3} v_{j} v_{k}} A_{i}(t)\left[\mathcal{E}_{\mathrm{F}}^{2}-\frac{e^{2}}{5} \sum_{l} v_{l}^{2} A_{l}^{2}(t)\right],
$$

where $j, k$ are such that $i \neq j \neq k$, and $l \in\{x, y, z\}$. When $\Phi(t)>\Phi_{\text {crit }}$, the current becomes

$$
\begin{aligned}
J_{i}^{3 \mathrm{D}, \text { sup }}(t)= & -\frac{g e \mathcal{E}_{\mathrm{F}}^{3}}{6 \pi^{2} \hbar^{3} v_{j} v_{k}} \\
& \times \frac{v_{i} A_{i}(t)}{\sqrt{\sum_{l} v_{l}^{2} A_{l}^{2}(t)}}\left[1-\frac{\mathcal{E}_{\mathrm{F}}^{2}}{5 e^{2} \sum_{l} v_{l}^{2} A_{l}^{2}(t)}\right] .
\end{aligned}
$$

Equations (2) and (3) are obtained in the low-temperature limit $(T \rightarrow 0 \mathrm{~K})$, assuming that the driving frequencies $\omega$ satisfy the relation $\hbar \omega \ll 2 \mathcal{E}_{\mathrm{F}}$ (intraband current dominates). Details of the derivation are presented in Appendix B 1 . Throughout this work, we term $\Phi_{\max }<\Phi_{\text {crit }}$ the subcritical regime and $\Phi_{\max }>\Phi_{\text {crit }}$ the supercritical regime, where $\Phi_{\max }$ is the maximum amplitude of $\Phi(t)$. Importantly, in the subcritical regime, only some electrons can be driven to the Dirac point; in the supercritical regime, all electrons can be driven past the Dirac point. As our theory is fully nonperturbative, Eqs. (2) and (3) contain every order of the conductivity. It is interesting to note that the first- and third-order conductivities extracted from Eq. (2) agree with existing expressions $[45,46]$ derived perturbatively from the Boltzmann equation. We note that we present the full, nonperturbative response of 3D DSMs, given by Eqs. (2) and (3), which is valid for arbitrarily strong driving fields, in closed form.

\section{SUPERCRITICAL REGIME OF NONLINEAR OPTICS}

The HHG energy spectrum shown in Fig. 1(b) indicates that extremely efficient intraband HHG up to the 31st order and beyond can be generated at modest driving laser powers. We consider a 250 -nm-thick $\mathrm{Cd}_{3} \mathrm{As}_{2}$ thin film (readily grown using molecular beam epitaxy [31,32,47-51]) of Fermi level $\mathcal{E}_{\mathrm{F}}=60 \mathrm{meV}$, driven by a normally incident, 2-ps-long pulse of peak frequency $1 \mathrm{THz}$ and peak field amplitude $E_{0, x}=10$ $\mathrm{MV} / \mathrm{m}$ [Fig. 1(b) inset] — routinely achievable using existing table-top THz sources [52-56]. We use experimentally verified values of the Fermi velocities for $\mathrm{Cd}_{3} \mathrm{As}_{2}:\left(v_{x}, v_{y}, v_{z}\right)=$ $(1.28,1.3,0.33) \times 10^{6} \mathrm{~m} / \mathrm{s}$ [57]. We find that the $31 \mathrm{st}$ harmonic of the output energy spectral density lies within five orders of magnitude of the fundamental harmonic. This compares favorably with the performance of conventional solid-state THz HHG, where the 22nd harmonic lies within eight orders of magnitude of the fundamental harmonic, when using input intensities $10^{5}$ times stronger than what we consider here [18]. When we operate deep in the supercritical regime $\Phi_{\max } \gg \Phi_{\text {crit }}$, Eq. (3) shows that the intraband current approaches $J_{x}^{\text {BD,sup }}(t) \rightarrow-\operatorname{sgn}\left[A_{x}(t)\right] g e \mathcal{E}_{\mathrm{F}}^{3} /\left(6 \pi^{2} \hbar^{3} v_{y} v_{z}\right)$, which describes a square-wave temporal profile containing only odd-ordered frequency components.

Figures $1(\mathrm{c})-1(\mathrm{~g})$ show that efficient HHG performance at extreme harmonics can still be accessed even at more modest field strengths. In Fig. 1, we consider $T=0 \mathrm{~K}$ and no carrier scattering. As shown in Appendix C, the HHG efficiency persists for larger temperatures and finite scattering, so long as the time taken to accelerate electrons from the Fermi surface past the Dirac point is shorter than the scattering time [31].

Although circularly and elliptically polarized driving pulses can be used to generate high-order harmonics, we find that linearly polarized driving light, as we consider here, yields the highest number of efficiently excited harmonics (Appendix D). Incidentally, we define the energy conversion efficiency in this work as $U_{N} / U_{\text {in }}$, where $U_{\text {in }}$ is the incident laser energy (considering only the part of the pulse that interacts with the sample) and $U_{N}$ is the output energy of the $N$ th harmonic.

\section{SUBCRITICAL REGIME OF NONLINEAR OPTICS}

In addition to the above regime of extreme nonlinearity, we also uncover a regime in 3D DSMs where higher order light emission beyond the third harmonic vanishes. As we see from Eq. (2), the emission due to the intraband current-which we term intraband emission-in the subcritical regime $\left(\Phi_{\max }<\right.$ $\Phi_{\text {crit }}$ ) exactly contains only the first and third harmonics with no higher orders [Fig. 2(a)]. This is a noteworthy feature of 3D DSMs, especially because 2D DSMs exhibit no such vanishing of HHG under any condition [Fig. 2(b)]. To further illustrate this, the emission spectra of 3D and 2D DSMs in the subcritical regime is shown in Figs. 2(c), 2(e) and Figs. 2(d), 2(f), respectively. This difference in 3D and 2D DSM behavior can also be seen by comparing the intraband current for $2 \mathrm{D}$ DSMs, given by Eq. (B22) in Appendix B 2-which contains every order of nonlinearity - with the intraband current for 3D DSMs in Eq. (2).

The reason why higher-order intraband nonlinearities in 3D DSM vanish is geometrical. Because of the extra dimension that 3D DSMs possess over 2D DSMs, the integral in Eq. (1) takes place over a spherical region in momentum space for 3D DSMs [Fig. 2(g)], in contrast to a circular region for 2D DSMs [Fig. 2(h)]. As the intraband contribution to the integrand for 3D DSMs can be recast as a sum of Legendre polynomials, and noting that higher order Legendre polynomials (associated with higher powers in the vector potential) vanish upon integration over the spherical domain, it follows that the higher order intraband nonlinearities also vanish (Appendix E).

In Fig. 2, we consider a temperature $T=0 \mathrm{~K}$ and no carrier scattering. However, we find that even when finite temperatures and carrier scattering effects are non-negligible, the suppression of higher order light emission remains significant (Appendix F). Furthermore, we note that this suppression of HHG in the subcritical regime of 3D DSMs is a phenomenon 

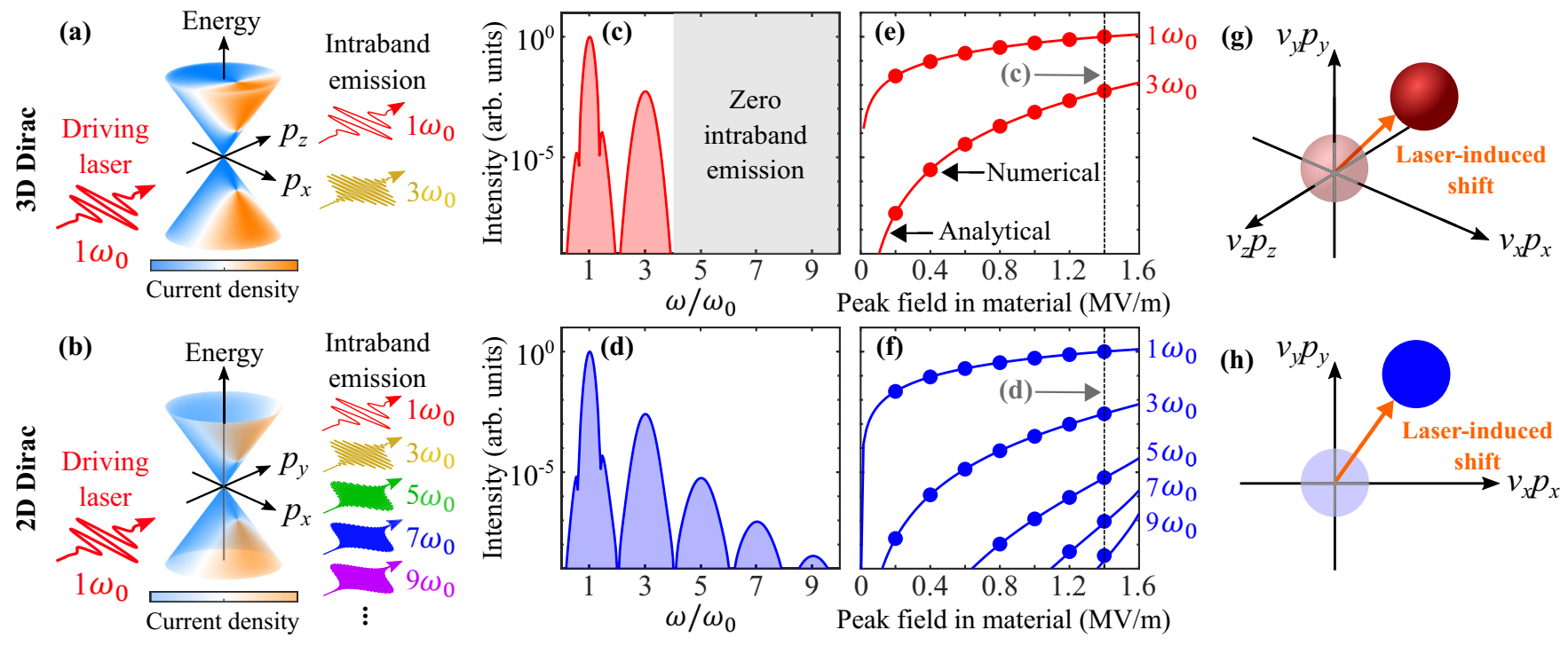

FIG. 2. Vanishing of the high-order intraband emission from 3D DSMs in the subcritical regime ( $\left.\Phi_{\max }<\Phi_{\text {crit }}\right)$. An incident laser field induces oscillating carriers in 3D Dirac cones (a) and 2D Dirac cones (b). However, the emission profile resulting from these oscillations differs drastically between the 3D case and the 2D case, breaking the notion that 3D DSMs are merely bulk versions of 2D DSMs. While optical nonlinearities abruptly disappear beyond the third order in 3D DSMs (c), they persist at every order in 2D DSMs (d). This phenomenon holds across a broad range of field strengths, as shown in panels (e) and (f). The contribution of the interband current is very weak and has been verified to fall below the intensity range displayed. In panels (e) and (f), we also see that the results of our fully closed-form, nonperturbative expressions (curves) are in excellent agreement with rigorous numerical simulations (solid circles). The reason why higher order nonlinearities vanish in 3D DSMs and not in 2D DSMs lies in the extra dimension that 3D DSMs possess compared to 2D DSMs. Despite sharing the same expression for the current [Eq. (1)], the required integration over 3D momentum space (g) for 3D DSMs, in contrast to integration over 2D momentum space (h) for 2D DSMs, leads to very different behavior in these materials. In panels (g) and (h), the colored solid areas represent the regions of integration, which are shifted by the applied laser field. In this comparison, we consider Fermi velocities $v_{x}=v_{y}=v_{z}=10^{6}$ $\mathrm{m} / \mathrm{s}$, Fermi energy $\mathcal{E}_{\mathrm{F}}=250 \mathrm{meV}$ at temperature $T=0 \mathrm{~K}$, and no carrier scattering.

that is robust against variations in driving field polarization, driving field phase, and Fermi velocity anisotropy (Appendix D).

\section{COMPARING VARIOUS 3D AND 2D DSMs}

In Fig. 3, we explore HHG in both subcritical and supercritical regimes for the experimentally realized 3D DSMs $\mathrm{Cd}_{3} \mathrm{As}_{2}$ [57] and $\mathrm{Na}_{3} \mathrm{Bi}$ [58]. We use experimentally obtained Fermi velocities for $\mathrm{Na}_{3} \mathrm{Bi}: \quad\left(v_{x}, v_{y}, v_{z}\right)=(4.17,3.63,0.95) \times$ $10^{5} \mathrm{~m} / \mathrm{s}$ [58], and, unless otherwise specified, the same parameters as in Fig. 2. Once again, we observe excellent agreement between our numerical spectra (markers) and closed-form expressions given by Eqs. (2) and (3) (curves). In the supercritical regime, we observe that 3D DSMs behave in a qualitatively similar manner as graphene [41,44,59], although we stress that $\mathrm{Cd}_{3} \mathrm{As}_{2}$ can surpass graphene by more than 100 times in output intensity under similar input conditions (Appendix G). Additionally, we note that Fermi velocity anisotropy plays a role in determining the nonlinear optical response in 3D DSMs, as we show through an example in Appendix $\mathrm{H}$.

\section{DISCUSSION}

Crucially, our theory reveals the existence of a unique regime in which highly efficient $\mathrm{THz} \mathrm{HHG}(\geqslant 29$ th harmonic) is possible (Fig. 4, upper right quadrant). This regime is distinct and complementary to recent experiments that have demonstrated efficient generation up to the third [31] and seventh [32] harmonics. While Ref. [31] explores a regime corresponding to slower scattering times $\omega_{0} \tau$ and weakly supercritical potential amplitudes $\Phi_{\max } / \Phi_{\text {crit }}$ (Fig. 4, upper left quadrant) and Ref. [32] investigates another regime corresponding to fast $\omega_{0} \tau$ and very supercritical $\Phi_{\max } / \Phi_{\text {crit }}$ (Fig. 4 , lower right quadrant), we find that a combination of slow $\omega_{0} \tau$ and very supercritical $\Phi_{\max } / \Phi_{\text {crit }}$ grants access to the novel-and only_regime in which it is possible to efficiently generate extreme $\mathrm{THz}$ harmonics up to the $31 \mathrm{st}$ order and beyond. From $\Phi_{\max } / \Phi_{\text {crit }}=e v_{x} E_{0, x} /\left(\omega_{0} \mathcal{E}_{\mathrm{F}}\right)$ for $x$-polarized driving light, we see that in addition to increasing $E_{0, x}$, moving to longer driving wavelengths can also increase the degree of supercriticality. The discovery of this regime of extreme nonlinearity takes us beyond previous works that demonstrate the generation of lower order harmonics and paves the way to realizing the full potential of 3D DSMs as efficient on-chip radiation sources for optoelectronics (see Appendix I for parameters used to plot Fig. 4 and a discussion on the number of harmonics predicted for the individual cases marked in Fig. 4).

\section{SUMMARY}

In summary, we show that 3D DSMs can efficiently generate extreme $\mathrm{THz}$ harmonics up to the 31st order and beyond using modest laser intensities $\approx 10 \mathrm{MW} / \mathrm{cm}^{2}$, $10^{5}$ times weaker than in conventional solid-state $\mathrm{HHG}$ 


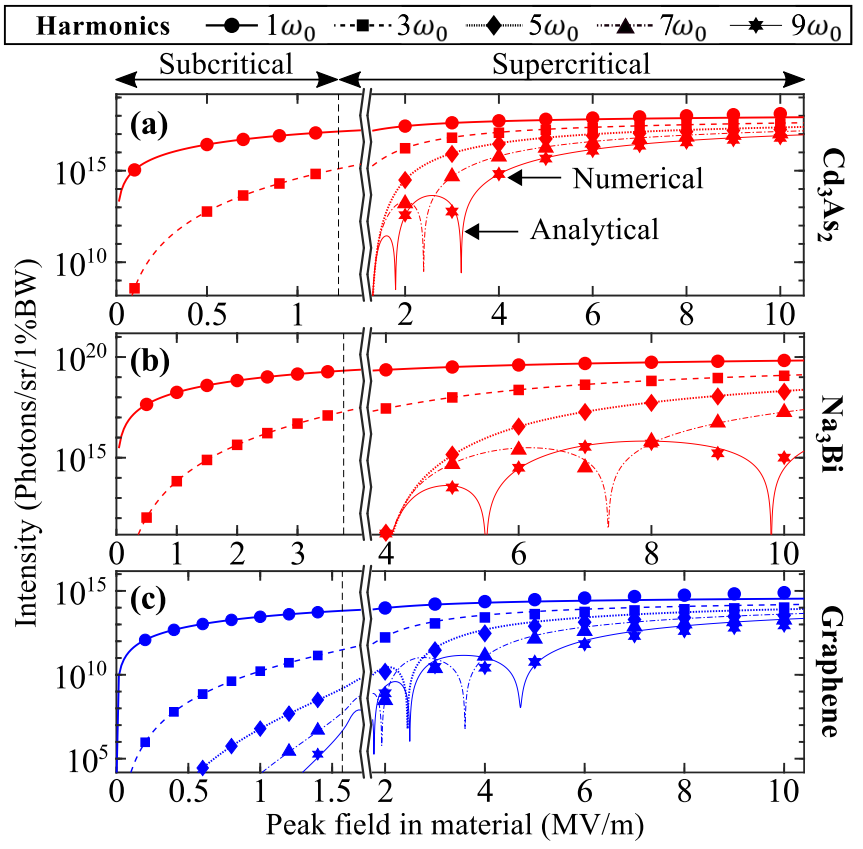

FIG. 3. High-order harmonic generation in the 3D DSM materials $\mathrm{Cd}_{3} \mathrm{As}_{2}$ (a) and $\mathrm{Na}_{3} \mathrm{Bi}$ (b), and in 2D DSM graphene (c). In the subcritical regime (field corresponding to critical potential marked by vertical black dashes), emitted harmonics beyond the third order are greatly suppressed in 3D DSMs. No such suppression occurs in 2D DSMs. In the supercritical regime, however, the intensity of the emitted harmonics in 3D DSMs rapidly increase with increasing field strength. In each panel, markers and lines denote numerical (intraband and interband emission) and analytical (intraband emission only) results, respectively. The good agreement between them indicates the dominance of intraband emission in our regime of interest. For the sake of clarity, we plot only up to the ninth harmonic.

[17-22,24-26]. Our fully closed-form, analytical expressions for the nonperturbative optical response of 3D DSMs, introduced through Eqs. (2) and (3), reveal that such highly efficient generation of extreme $\mathrm{THz}$ harmonics is only possible within a regime of extreme nonlinearity. This distinct regime is complementary to recent experiments demonstrating the efficient generation of lower order $\mathrm{THz}$ harmonics [31,32] from 3D DSMs. Additionally, we unveil a subcritical regime in which intraband nonlinearities beyond the third order completely vanish, resulting in greatly suppressed higher harmonic output. We further show that this vanishing of intraband emission arises geometrically from the extra dimensionality of 3D DSMs compared to 2D DSMs, breaking the common notion that 3D DSMs share the essential physics of 2D DSMs. Our work fills a vital gap in the understanding of nonlinear optics in 3D DSMs, paving the way to unlocking the full potential of 3D DSMs as highly efficient, chip-integrable $\mathrm{THz}$ light sources for optoelectronics.

\section{ACKNOWLEDGMENTS}

L.J.W. acknowledges the support of the Agency for Science, Technology and Research (A*STAR) Advanced Manufacturing and Engineering Young Individual Research Grant (No. A1984c0043), and the Nanyang Assistant Profes-

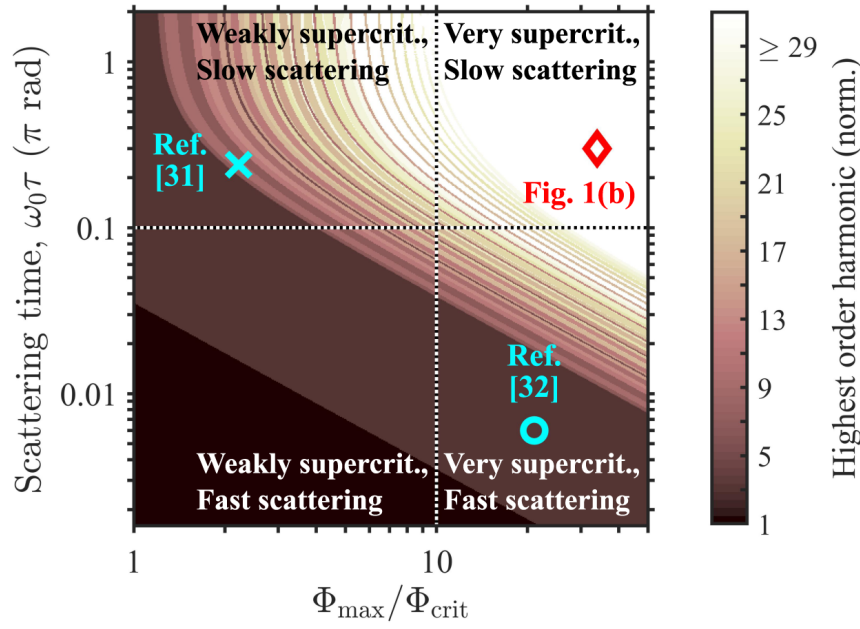

FIG. 4. Existence of a unique operation regime (upper right quadrant) in which highly efficient generation of extreme $\mathrm{THz}$ harmonics $(\geqslant 29$ th orders) is possible. Our theory reveals that this distinct regime is complementary to existing demonstrations of harmonic generation of up to the third [31] (cyan cross) and seventh [32] (cyan circle) order: By combining a potential amplitude $\Phi_{\max } / \Phi_{\text {crit }}$ similar to that used in Ref. [32], and a scattering time $\omega_{0} \tau$ similar to that of Ref. [31], the extreme nonlinearity that becomes possible only within this regime can be leveraged for highly efficient THz HHG up to the 31st order and beyond, as we predict in Fig. 1(b) (red diamond). The color map is computed using Eqs. (2) and (3) at $T=0 \mathrm{~K}$. Only normalized harmonic intensities $\geqslant 10^{-6}$ are considered.

sorship Start-up Grant. J.L., Y.S.A., and L.K.A. acknowledge funding from Singapore Ministry of Education (MOE) Tier 2 Grant (No. 2018-T2-1-007), MOE PhD RSS, and USA Office of Naval Research Global (ONRG) Grant (No. N62909-19-1-2047). I.K. acknowledges the support of the Azrieli Faculty Fellowship, the Israel Science Foundation Grants No. 3334/19 and No. 831/19, and the ERC Starting Grant NanoEP 851780 from the European Research Council. F.J.G.d.A acknowledges funding from ERC (Advanced Grant No. 789104-eNANO) and Spanish MINECO (Grants No. MAT2017-88492-R and No. SEV2015-0522). Numerical work was partially performed on the computational resources provided by the National Supercomputing Centre Singapore (NSCC).

\section{APPENDIX A: METHODS}

\section{Nonperturbative massless electron dynamics}

Here, we derive the ordinary differential equations (ODE) governing the coupled behavior of the interband and intraband electron dynamics in a 3D DSM. Our derivation follows from the time-dependent Dirac equation (TDDE) in the main text. Using the minimal coupling substitution, the low-energy Hamiltonian reads

$$
i \hbar \frac{\partial}{\partial t}=\hat{\mathcal{H}}_{\mathbf{p}}=\sum_{j} v_{j} \sigma_{j} \pi_{j}(t),
$$

where $j \in\{x, y, z\}$ runs over Cartesian directions, $\hbar$ is the reduced Planck constant, $v_{j}$ are the Fermi velocities associated with directions $j, \sigma_{j}$ are the Pauli matrices, $\pi_{j}(t)=p_{j}+$ 
$e a_{j}(t)$ are the components of the modified minimal coupling momentum, $p_{j}$ is the unperturbed electron momentum, $e$ is the elementary change, and $a_{j}(t)$ is defined as [44]

$$
a_{j}(t)=-e^{-t / \tau} \int_{-\infty}^{t} E_{j}\left(t^{\prime}\right) e^{t^{\prime} / \tau} d t^{\prime}
$$

with $\tau$ being a phenomenological inelastic intraband scattering time and $E_{j}(t)$ being the time-varying, spatially uniform electric field. In the absence of intraband scattering (i.e., $\tau \rightarrow$ $\infty), a_{j}(t)$ is exactly equivalent to the unmodified vector potential $A_{j}(t)=-\int_{-\infty}^{t} E_{j}\left(t^{\prime}\right) d t^{\prime}$. The fields should include the self-consistent response of the material, but for simplicity we approximate it as the external field, neglecting induced-field effects. Following previous studies on graphene $[41,42,44]$, we write the normalized, instantaneous eigenstates as

$$
\begin{aligned}
& \psi_{\mathbf{p}, \mathrm{c}}(t)=\left\{\begin{array}{l}
\cos [\theta(t) / 2] e^{-i \phi(t) / 2} \\
\sin [\theta(t) / 2] e^{+i \phi(t) / 2}
\end{array}\right\} e^{-i \Omega(t)}, \\
& \psi_{\mathbf{p}, \mathrm{v}}(t)=\left\{\begin{array}{c}
\sin [\theta(t) / 2] e^{-i \phi(t) / 2} \\
-\cos [\theta(t) / 2] e^{+i \phi(t) / 2}
\end{array}\right\} e^{+i \Omega(t)} .
\end{aligned}
$$

The "c" and "v" subscripts denote conduction and valence band states respectively. We further define $\theta(t)=$ $\arccos \left[v_{z} \pi_{z}(t) / \mathcal{E}(t)\right]$ and $\phi(t)=\arctan \left[v_{y} \pi_{y}(t) / v_{x} \pi_{x}(t)\right]$. The dynamical phase is $\Omega(t)=\hbar^{-1} \int_{t_{0}}^{t} \mathcal{E}\left(t^{\prime}\right) d t^{\prime}$, where $\mathcal{E}(t)=$ $\sqrt{v_{x}^{2} \pi_{x}^{2}+v_{y}^{2} \pi_{y}^{2}+v_{z}^{2} \pi_{z}^{2}}$ represents the instantaneous energy. The most general wave function that satisfies Eq. (A1) can be constructed by superposing the above eigenstates:

$$
\Psi_{\mathbf{p}}(t)=C_{\mathbf{p}, \mathrm{c}}(t) \psi_{\mathbf{p}, \mathrm{c}}(t)+C_{\mathbf{p}, \mathrm{v}}(t) \psi_{\mathbf{p}, \mathrm{v}}(t) .
$$

The complex coefficients $C_{\mathbf{p}, \mathrm{c}}(t)$ and $C_{\mathbf{p}, \mathrm{v}}(t)$ describe how the probability amplitude for finding the electron in either state evolves with time. By using Eq. (A4) as an ansatz to the Schödinger equation [i.e., $\left.i \hbar \partial_{t} \Psi_{\mathbf{p}}(t)=\hat{\mathcal{H}}_{\mathbf{p}} \Psi_{\mathbf{p}}(t)\right]$ and defining the population inversion (difference of electron population between the valence and conduction bands), $\mathcal{N}_{\mathbf{p}}(t)=$ $\left|C_{\mathbf{p}, \mathrm{c}}(t)\right|^{2}-\left|C_{\mathbf{p}, \mathrm{v}}(t)\right|^{2}$, and the interband coherence, $\Gamma_{\mathbf{p}}(t)=$ $\left[C_{\mathbf{p}, \mathrm{v}}(t)\right]^{*} C_{\mathbf{p}, \mathrm{c}}(t) e^{-2 i \Omega(t)}$, where ${ }^{*}$ denotes the complex conjugate, we can derive a set of ODEs, which describe how these quantities vary in time:

$$
\begin{aligned}
\dot{\mathcal{N}}_{\mathbf{p}}(t)= & -\frac{1}{\tau}\left[\mathcal{N}_{\mathbf{p}}(t)-\mathcal{N}_{\mathbf{p}}\left(t_{0}\right)\right]-2 \dot{\theta}(t) \operatorname{Re}\left[\Gamma_{\mathbf{p}}(t)\right] \\
& +2 \dot{\phi}(t) \sin \theta(t) \operatorname{Im}\left[\Gamma_{\mathbf{p}}(t)\right] \\
\dot{\Gamma}_{\mathbf{p}}(t)= & {\left[i \dot{\phi}(t) \cos \theta(t)-\frac{1}{\tau}-\frac{2 i \mathcal{E}(t)}{\hbar}\right] \Gamma_{\mathbf{p}}(t) } \\
& +[\dot{\theta}(t)-i \dot{\phi}(t) \sin \theta(t)] \frac{\mathcal{N}_{\mathbf{p}}(t)}{2}
\end{aligned}
$$

Here, inelastic interband damping has been introduced phenomenologically through the scattering time $\tau$. While $\tau$ in Eqs. (A5a) and (A5b) can be in principle different from $\tau$ in Eq. (A2), we use the same value for both scattering times. At some initial time $t_{0}$, long before the laser pulse interacts with the electrons, we have $\mathcal{N}_{\mathbf{p}}\left(t_{0}\right)=f_{\mathrm{D}}\left[\mathcal{E}\left(t_{0}\right)\right]-f_{\mathrm{D}}\left[-\mathcal{E}\left(t_{0}\right)\right]$ and $\Gamma_{\mathbf{p}}\left(t_{0}\right)=0$, where $f_{\mathrm{D}}(\mathcal{E})=\left(1+\exp \left\{[\mathcal{E}-\mu(T)] / k_{\mathrm{B}} T\right\}\right)^{-1}$ is the Fermi-Dirac distribution for a chemical potential $\mu(T)$ at temperature $T$ and $k_{\mathrm{B}}$ is the Boltzmann constant. Equations (A5a) and (A5b) describe how a massless electron moves within (intraband) and transitions between (interband) energy bands under the influence of driving fields, unlike the semiclassical Boltzmann equation, which only describes intraband motion. In deriving these ODEs, we have made no approximations beyond a constant scattering time and the massless electron limit, as well as incorporating Hartree interactions within a linear response through the procedure explained below. The equivalent equations for the $2 \mathrm{D}$ case, which have been successful in describing infinitely extended graphene interacting with a laser $[41,42,44]$, are obtained by neglecting the $z$ components and setting $\theta=\pi / 2$, effectively limiting interactions to within the $p_{x}-p_{y}$ plane.

The induced current due to a single momentum value $\mathbf{p}$ is computed as

$$
\mathbf{j}_{\mathbf{p}}(t)=-e \Psi_{\mathbf{p}}^{\dagger}(t) \nabla_{\pi} \hat{\mathcal{H}}_{\mathbf{p}} \Psi_{\mathbf{p}}(t),
$$

where $\nabla_{\pi} \hat{\mathcal{H}}_{\mathbf{p}}=\left(v_{x} \sigma_{x}, v_{y} \sigma_{y}, v_{z} \sigma_{z}\right)$ is the group velocity operator and the $\dagger$ supercript denotes Hermitian conjugation. The individual current components are

$$
\begin{gathered}
j_{\mathbf{p}, x}=-e v_{x}\left\{\mathcal{N}_{\mathbf{p}} \sin \theta \cos \phi-2 \cos \theta \cos \phi \operatorname{Re}\left(\Gamma_{\mathbf{p}}\right)\right. \\
\left.-2 \sin \phi \operatorname{Im}\left(\Gamma_{\mathbf{p}}\right)\right\}, \\
j_{\mathbf{p}, y}=-e v_{y}\left\{\mathcal{N}_{\mathbf{p}} \sin \theta \sin \phi-2 \cos \theta \sin \phi \operatorname{Re}\left(\Gamma_{\mathbf{p}}\right)\right. \\
\left.\quad+2 \cos \phi \operatorname{Im}\left(\Gamma_{\mathbf{p}}\right)\right\}, \\
j_{\mathbf{p}, z}=-e v_{z}\left\{\mathcal{N}_{\mathbf{p}} \cos \theta+2 \sin \theta \operatorname{Re}\left(\Gamma_{\mathbf{p}}\right)\right\} .
\end{gathered}
$$

The total induced current is obtained by integrating over all momentum space:

$$
\mathbf{J}(t)=\frac{g}{(2 \pi \hbar)^{3}} \iiint \mathbf{j}_{\mathbf{p}}(t) d^{3} \mathbf{p},
$$

where $g$ is the electron state degeneracy (i.e., number of Dirac cones in the first Brillouin zone times spin degeneracy) and the integral extends over the entire momentum space. When performing the integral in Eq. (A8), the $\mathcal{N}_{\mathbf{p}}$ terms in Eqs. (A7a)-(A7c) must replaced by $\mathcal{N}_{\mathbf{p}}+1$, as part of a regularization procedure to prevent divergences at large momenta due to the assumption of an infinitely extended Dirac cone $[41,42,44,60]$. Equation (A8), with the integrand represented by Eqs. (A7a)-(A7c), is equivalent to Eq. (1) in the main text.

\section{Nonperturbative time-domain quantum simulations}

Equations (A5a) and (A5b) are discretized on a scaled momentum space grid $\left(q_{i}=v_{i} p_{i}\right.$, where $\left.i \in\{x, y, z\}\right)$. We then numerically integrate them over time using the DormandPrince adaptive step solver (Boost $\mathrm{C}++$ library) [61]. The single-electron currents are integrated over all momentum space using the trapezoidal rule.

While simulations of graphene and other 2D DSMs are manageable on a discretized momentum grid, the simulations quickly become intractible due to the additional dimension in 3D Dirac semimetals. When the incident laser is linearly polarized, we can exploit cylindrical symmetry by aligning the polarization axis parallel to $q_{z}=v_{z} p_{z}$, effectively reducing the 
problem to 2D. Equations (A5a) to (A5b) then become

$$
\begin{aligned}
& \dot{\mathcal{N}}_{\mathbf{p}}(t)=-2 \dot{\theta}(t) \operatorname{Re}\left[\Gamma_{\mathbf{p}}(t)\right]-\frac{1}{\tau}\left[\mathcal{N}_{\mathbf{p}}(t)-\mathcal{N}_{\mathbf{p}}\left(t_{0}\right)\right], \\
& \dot{\Gamma}_{\mathbf{p}}(t)=\frac{1}{2} \dot{\theta}(t) \mathcal{N}_{\mathbf{p}}(t)-\left[\frac{1}{\tau}+\frac{2 i \mathcal{E}(t)}{\hbar}\right] \Gamma_{\mathbf{p}}(t) .
\end{aligned}
$$

The time derivatives of the angular terms then become $\dot{\phi}=0$ and $\dot{\theta}(t)=e v_{z} E_{z}(t) q_{\rho} / \mathcal{E}(t)^{2}$, where $E_{z}(t)$ is the electric field component along $z$ and $q_{\rho}$ is the radial coordinate in the scaled momentum space. Note that the above equations and the initial conditions have no angular dependence, which is also the case for the single-electron current:

$$
j_{\mathbf{p}, z}(t)=-e v_{z}\left\{\frac{\left[\mathcal{N}_{\mathbf{p}}(t)+1\right]\left(q_{z}+e v_{z} A_{z}\right)+2 q_{\rho} \operatorname{Re}\left[\Gamma_{\mathbf{p}}(t)\right]}{\sqrt{\left(q_{z}+e v_{z} A_{z}\right)^{2}+q_{\rho}^{2}}}\right\} .
$$

Since both the single-electron current and Eqs. (A9a) and (A9b) are rotationally invariant around the axis of polarization, we have effectively reduced the $3 \mathrm{D}$ problem to $2 \mathrm{D}$. Thus, for linearly polarized illumination, we compute the macroscopic current along $z$, integrated over all momentum-resolved contributions as

$$
J_{z}(t) \propto 2 \pi \int_{0}^{+\infty} \int_{-\infty}^{+\infty} j_{\mathbf{p}, z}(t) q_{\rho} d q_{z} d q_{\rho} .
$$

\section{Modeling ultrafast THz pulses}

High-intensity THz pulses from compact sources are frequently realized in the few-to-single cycle regime. To simulate a pulsed plane wave that does not contain spurious nonzero DC components, we choose a Poisson power spectrum, which accurately describes ultrafast pulses with durations down to single laser period [62]:

$$
F(\omega)=2 \pi e^{i \phi_{0}}\left(\frac{s}{\omega_{0}}\right)^{s+1} \frac{\omega^{s} \exp \left(-s \omega / \omega_{0}\right)}{\Gamma(s+1)} \Theta(\omega) .
$$

Here, $\omega$ is angular frequency, $\phi_{0}$ is a phase constant, $s$ is a real, positive parameter that determines the pulse duration, $\omega_{0}$ is the peak angular frequency of the pulse, $\Gamma$ is the gamma function, and $\Theta$ is the Heaviside step function. In the narrow bandwidth limit (i.e., the many-cycle limit), Eq. (A12) approaches a Gaussian spectrum of central angular frequency $\omega_{0}$. The time-dependent electric field whose spectrum is given by Eq. (A12) reads

$$
\mathbf{E}(t)=\operatorname{Re}\left\{\mathbf{E}_{0} e^{i \phi_{0}}\left[1+\frac{i \omega_{0}\left(t-t_{\mathrm{pk}}\right)}{s}\right]^{-s-1}\right\},
$$

where $\mathbf{E}_{0}=\left(E_{x, 0}, E_{y, 0}, E_{z, 0}\right)$ is the peak amplitude and $t_{\mathrm{pk}}$ is the time at which the pulse reaches its peak. The intensity full width at half maximum (FWHM) $\tau_{\mathrm{FWHM}}$ is related to parameter $s$ via $\tau_{\mathrm{FWHM}}=2 s \sqrt{2 \ln (2) /(2 s-1)} / \omega_{0}$. Throughout this work, all pulse durations refer to the intensity FWHM. For $\tau_{\mathrm{FWHM}}=2 \mathrm{ps}$, the corresponding shape factor is $s \approx 56.4$. We also use the vector potential $\mathbf{A}(t)=\left[A_{x}(t), A_{y}(t), A_{z}(t)\right]$ at time $t$, which is given by

$$
\mathbf{A}(t)=-\int_{-\infty}^{t} \mathbf{E}\left(t^{\prime}\right) d t^{\prime} .
$$

\section{THz interaction with 3D DSM thin film}

To model the interaction of a linearly polarized driving $\mathrm{THz}$ pulse with a finite 3D DSM thin film at normal incidence, we employ a $1+1 \mathrm{D}$ finite-difference time-domain (FDTD) routine that fully incorporates all intraband nonlinearities induced by the incident field. At the starting location $z=z_{\text {in }}$, we have the input fields $\mathbf{E}_{\text {in }}=\left(E_{\text {in }, x}, 0,0\right)$ and $\mathbf{H}_{\text {in }}=$ $\left(0, E_{\mathrm{in}, x} / \eta_{0}, 0\right)$, where $\eta_{0}$ is the free space impedance and $E_{\mathrm{in}, x}$ is given by

$$
E_{\mathrm{in}, x}\left(z_{\mathrm{in}}, t\right)=\operatorname{Re}\left\{E_{x, 0} e^{i \phi_{0}}\left[1-\frac{i\left(k_{0} z^{\prime}-\omega_{0} t\right)}{s}\right]^{-s-1}\right\},
$$

where $k_{0}=\omega_{0} / c$ is the free-space light wave number. We define $z^{\prime}=z_{\mathrm{in}}-z_{\mathrm{pk}}$, where $z_{\mathrm{pk}}$ is the initial pulse peak location. We write Maxwell's equations in the form

$$
\begin{aligned}
\partial_{t} H_{y} & =-\frac{1}{\mu} \partial_{z} E_{x}, \\
\epsilon_{0} \partial_{t} E_{x} & =-\partial_{z} H_{y}-J_{x} .
\end{aligned}
$$

The total current is the sum $J_{x}=J_{x}^{\mathrm{fr}}+J_{x}^{\mathrm{pol}}$, where $J_{x}^{\mathrm{fr}}$ is the free current and $J_{x}^{\mathrm{pol}}=\partial_{t} P_{x}$ is the current arising from the dielectric polarization $P_{x}$. To evaluate $J_{x}$, we use our analytical solutions for the intraband current, given by Eqs. (2) and (3) in the main text.

We discretize these equations on a Yee grid, which is staggered in both time and space, for the evaluation of both the electric and magnetic fields. Defining normalized time and spatial steps $\Delta\left(\omega_{0} t\right)$ and $\Delta\left(k_{0} z\right)$ respectively, we obtain

$$
\begin{aligned}
\left.c B_{y}\right|_{j+1 / 2} ^{n+1 / 2}= & \left.c B_{y}\right|_{j+1 / 2} ^{n-1 / 2}-\frac{\Delta\left(\omega_{0} t\right)}{\Delta\left(k_{0} z\right)}\left(\left.E_{x}\right|_{j+1} ^{n}-\left.E_{x}\right|_{j} ^{n}\right),(\mathrm{A} 17 \mathrm{a}) \\
\left.E_{x}\right|_{j} ^{n+1}= & \left.E_{x}\right|_{j} ^{n}-\frac{1}{\mu_{r}} \frac{\Delta\left(\omega_{0} t\right)}{\Delta\left(k_{0} z\right)}\left(\left.c B_{y}\right|_{j+1 / 2} ^{n+1 / 2}-\left.c B_{y}\right|_{j-1 / 2} ^{n+1 / 2}\right) \\
& -\left.\frac{\Delta\left(\omega_{0} t\right)}{\omega_{0} \epsilon_{0}} J_{x}\right|_{j} ^{n+1} .
\end{aligned}
$$

We have assumed that the material is linear and homogeneous in magnetic field response: $B_{y}=\mu_{0} \mu_{r} H_{y}\left(\mu_{r}=1\right.$ throughout this work). The upper index $n$ denotes the time step. The lower index refers to the spatial grid position. We also implement Mur absorbing boundary conditions [63] at both ends of our FDTD grid.

We note that $\left.J_{x}\right|_{j} ^{n+1}$ makes this scheme implicit because the current depends on the field $\left.E_{x}\right|_{j} ^{n+1}$ at the current time step. To obtain the correct $\left.J_{x}\right|_{j} ^{n+1}$ and $\left.E_{x}\right|_{j} ^{n+1}$, we employ a fixed-point iteration method in which $\left.J_{x}\right|_{j} ^{n+1}=0$ is used as an initial guess to compute $\left.E_{x}\right|_{j} ^{n+1}$. We use this first-pass solution to obtain a refined approximation of $\left.J_{x}\right|_{j} ^{n+1}$. This procedure is iterated until the error between two consecutively refined values of $\left.E_{x}\right|_{j} ^{n+1}$ are within a specified tolerance. We varied this tolerance, the values of $\Delta\left(k_{0} z\right)$, the Courant number $\Delta\left(\omega_{0} t\right) / \Delta\left(k_{0} z\right), z_{\mathrm{pk}}$, and simulation box width until convergence was achieved for the overall simulation. We assume free space on either side of the 3D DSM film, although this algorithm can be readily adapted to account for the presence of adjoining complex dispersive media (by incorporating the 


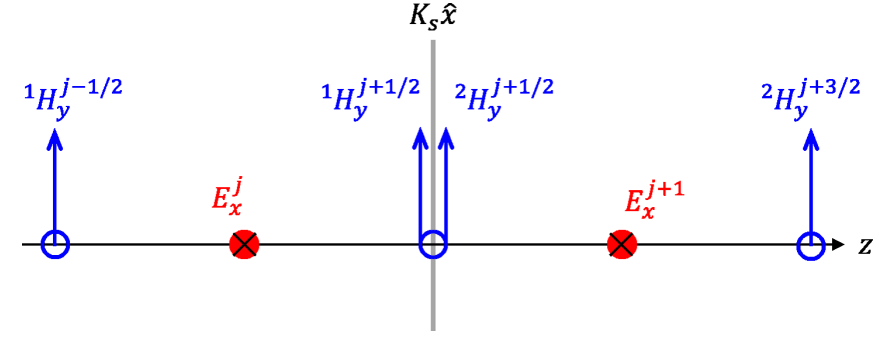

FIG. 5. Representation of the discretization method used in the SBC FDTD scheme for graphene. At spatial grid point $j+1 / 2$, where the graphene sheet is located, $H_{y}$ is evaluated on either side of the sheet.

implementation in Ref. [64]), or adjoining nonlinear media of other kinds whose behavior can be captured through the current $J_{x}$ as a function of $E_{x}$ like in Eqs. (2) and (3) in the main text.

We note that the use of a plane-wave input pulse and a 1+1D FDTD - as opposed to a focused laser pulse and a 3+1D FDTD - ignores effects arising from beam diffraction and wave-front curvature. However, it should be noted that for a weakly focused laser pulse (even one of high field strengths), a plane-wave pulse is a reasonable approximation.

\section{THz interaction with 2D DSM graphene}

Graphene has been modeled extensively within the FDTD framework as thin films. However, extremely fine discretization of the spatial grid $(<1 \mathrm{~nm})$ is required for convergence. As we are using incident wavelengths of the order of $100 \mu \mathrm{m}$, even a 1+1D FDTD routine quickly becomes computationally impractical. To appropriately capture the true 2D nature of graphene without resorting to the thin-film approximation, we adopt a 1D surface boundary condition (SBC) FDTD approach [65] that considers a surface current density instead of a volume current density. In this Appendix, we present our modification to this scheme, which incorporates the full nonlinear intraband response of the induced surface current.

We consider the same field configuration as laid out for the 3D DSM case above. Let $E_{x}$ be evaluated at all integer spatial grid points $\ldots, j-1, j, j+1, \ldots$, and $H_{y}$ be evaluated at all half-integer spatial grid points $\ldots, j-1 / 2, j+1 / 2, j+$ $3 / 2, \ldots$ (see Fig. 5). Let the graphene interface be located at spatial grid point $j+1 / 2$. Let $\left.{ }^{1} H_{y}\right|_{j+1 / 2} ^{n+1 / 2}$ and $\left.{ }^{2} H_{y}\right|_{j+1 / 2} ^{n+1 / 2}$ be the tangential fields on the left and right sides of the graphene sheet, respectively (evaluated at time point $n+1 / 2$ ). The interface conditions for the tangential $H$ fields yield

$$
\begin{aligned}
\hat{\mathbf{z}} & \times\left(\left.\mathbf{H}^{2}\right|_{j+1 / 2} ^{n+1 / 2}-\left.\mathbf{H}^{1}\right|_{j+1 / 2} ^{n+1 / 2}\right)=\left.\mathbf{K}_{s}\right|_{j+1 / 2} ^{n+1 / 2} \\
& \left.\Rightarrow{ }^{2} H_{y}\right|_{j+1 / 2} ^{n+1 / 2}-\left.{ }^{1} H_{y}\right|_{j+1 / 2} ^{n+1 / 2}=-\left.K_{s}\right|_{j+1 / 2} ^{n+1 / 2},
\end{aligned}
$$

where $\mathbf{K}_{s}=K_{s} \hat{\mathbf{x}}$ is the surface current induced within the graphene sheet. We discretize Faraday's law at $j+1 / 2$ using central differences in time. For media 1 and 2, we use backward and forward finite difference for $\partial_{z}$, leading to

$$
\begin{aligned}
\left.\mu_{1} \partial_{t}{ }^{1} H_{y}\right|_{j+1 / 2} & =-\left.\left.\partial_{z} E_{x}\right|_{j+1 / 2} \Rightarrow{ }^{1} H_{y}\right|_{j+1 / 2} ^{n+1 / 2} \\
& =\left.{ }^{1} H_{y}\right|_{j+1 / 2} ^{n-1 / 2}-\frac{2 \Delta t}{\mu_{1} \Delta z}\left[\left.E_{x}\right|_{j+1 / 2} ^{n}-\left.E_{x}\right|_{j} ^{n}\right]
\end{aligned}
$$

and

$$
\begin{aligned}
\left.\mu_{2} \partial_{t}{ }^{2} H_{y}\right|_{j+1 / 2} & =-\left.\left.\partial_{z} E_{x}\right|_{j+1 / 2} \Rightarrow{ }^{2} H_{y}\right|_{j+1 / 2} ^{n+1 / 2} \\
& =\left.{ }^{2} H_{y}\right|_{j+1 / 2} ^{n-1 / 2}-\frac{2 \Delta t}{\mu_{2} \Delta z}\left[\left.E_{x}\right|_{j+1} ^{n}-\left.E_{x}\right|_{j+1 / 2} ^{n}\right] .
\end{aligned}
$$

Notice that we need $\left.E_{x}\right|_{j+1 / 2} ^{n}$ but $E_{x}$ is not defined at grid point $j+1 / 2$. We have to use Eq. (A18), where $\left.K_{s}\right|_{j+1 / 2} ^{n}$ also depends on $\left.E_{x}\right|_{j+1 / 2} ^{n}$. From the interface condition given by Eq. (A18), we find

$$
\left.{ }^{2} H_{y}\right|_{j+1 / 2} ^{n}-\left.{ }^{1} H_{y}\right|_{j+1 / 2} ^{n}=-\left.K_{s}\right|_{j+1 / 2} ^{n} .
$$

However, $H_{y}$ is evaluated at time steps $n-1 / 2$ and $n+1 / 2$ instead of $n$. To estimate its value at time step $n$, we take the average of $\left.H_{y}\right|_{j+1 / 2} ^{n-1 / 2}$ and $\left.H_{y}\right|_{j+1 / 2} ^{n+1 / 2}$ :

$$
\begin{aligned}
& \left(\left.{ }^{2} H_{y}\right|_{j+1 / 2} ^{n+1 / 2}+\left.{ }^{2} H_{y}\right|_{j+1 / 2} ^{n-1 / 2}\right) \\
& \quad-\left(\left.{ }^{1} H_{y}\right|_{j+1 / 2} ^{n+1 / 2}+\left.{ }^{1} H_{y}\right|_{j+1 / 2} ^{n-1 / 2}\right)=-\left.2 K_{s}\right|_{j+1 / 2} ^{n} .
\end{aligned}
$$

Using Eqs. (A19) and (A20), this relation in $\left.K_{s}\right|_{j+1 / 2} ^{n}$ can be restated as

$$
\begin{aligned}
\left.E_{x}\right|_{j+1 / 2} ^{n}= & \left.C_{1} E_{x}\right|_{j+1} ^{n}+\left.C_{2} E_{x}\right|_{j} ^{n} \\
& +C_{3}\left[\left.{ }^{1} H_{y}\right|_{j+1 / 2} ^{n-1 / 2}-\left.{ }^{2} H_{y}\right|_{j+1 / 2} ^{n-1 / 2}-\left.K_{x}\right|_{j+1 / 2} ^{n}\right],
\end{aligned}
$$

where the constants are defined to be

$$
\begin{aligned}
C_{1} & =\left(\frac{\mu_{1}}{\mu_{1}+\mu_{2}}\right), \\
C_{2} & =\left(\frac{\mu_{2}}{\mu_{1}+\mu_{2}}\right), \\
C_{3} & =\frac{\Delta z}{\Delta t}\left(\frac{\mu_{1} \mu_{2}}{\mu_{1}+\mu_{2}}\right) .
\end{aligned}
$$

Now, we have an implicit equation for $\left.E_{x}\right|_{j+1 / 2} ^{n}$ and $\left.K_{s}\right|_{j+1 / 2} ^{n}$, which we iteratively solve using the same method we employed for the 3D DSM case until convergence is reached. To compute the intraband-only surface current $K_{s}$ without resorting to series expansions, we numerically evaluate the integral in Eq. (B18) using the $h$-adaptive integration routine in the Cubature $\mathrm{C}++$ library [66]. Upon obtaining converged values of $\left.E_{x}\right|_{j+1 / 2} ^{n}$, we update $\left.{ }^{1} H_{y}\right|_{j+1 / 2} ^{n+1 / 2}$ and $\left.{ }^{2} H_{y}\right|_{j+1 / 2} ^{n+1 / 2}$ via Eqs. (A19) and (A20). Then, $\left.{ }^{1} H_{y}\right|_{j+1 / 2} ^{n+1 / 2}$ and $\left.{ }^{2} H_{y}\right|_{j+1 / 2} ^{n+1 / 2}$ are used to update $\left.E_{x}\right|_{j+1}$ and $\left.E_{x}\right|_{j-1}$. For all other points away from the interface, $E_{x}$ and $H_{y}$ are updated using the conventional Yee grid scheme. Incidentally, we consider $\mu_{1}=\mu_{2}=\mu_{0}$ for the entire simulation domain. 


\section{Computing the far-field HHG spectra from DSMs}

Here, we derive the expression used to compute the farfield high-order harmonic spectra. We consider the expansion of the induced volume current density in the material:

$$
\mathbf{J}(\mathrm{r}, t)=\operatorname{Re}\left\{\frac{1}{2 \pi} \int \tilde{\mathbf{J}}(\mathbf{r}, \omega) e^{i \omega t} d \omega\right\} .
$$

The complex vector potential $\tilde{\mathbf{A}}(\mathbf{r}, \omega)$ that satisfies the Helmholtz equation

$$
\left(\nabla^{2}+\mu_{0} \epsilon_{0} \omega^{2}\right) \tilde{\mathbf{A}}(\mathbf{r}, \omega)=-\mu_{0} \tilde{\mathbf{J}}(\mathbf{r}, \omega)
$$

is

$$
\tilde{\mathbf{A}}(\mathbf{r}, \omega)=\frac{\mu_{0}}{4 \pi} \int \frac{\tilde{\mathbf{J}}(\mathbf{r}, \omega) e^{-i k r^{\prime \prime}}}{r^{\prime \prime}} d^{3} \mathbf{r}^{\prime},
$$

where $r^{\prime \prime}=\left|\mathbf{r}-\mathbf{r}^{\prime}\right|$ is the separation between observer position $\mathbf{r}$ and source position $\mathbf{r}^{\prime}$, and $k=\omega / c$ is the wave number. We consider the far-field region and expand $r^{\prime \prime}$ and $1 / r^{\prime \prime}$, retaining only terms which fall as $1 / r$. In this limit, the far-field vector potential is

$$
\tilde{\mathbf{A}}(\mathbf{r}, \omega) \approx \frac{\mu_{0} e^{-i k r}}{4 \pi r} \mathbf{N},
$$

where

$$
\mathbf{N}=\int \tilde{\mathbf{J}}(\mathbf{r}, \omega) e^{i k \mathbf{r}^{\prime} \cdot \hat{\mathbf{r}}} d^{3} \mathbf{r}^{\prime} .
$$

We use cylindrical source coordinates $\mathbf{r}^{\prime}=\left(\mathbf{r}_{\perp}^{\prime}, z^{\prime}\right)$, spherical observer coordinates, and assume that $\tilde{\mathbf{J}}\left(z^{\prime}, \omega\right)$ only depends on $z^{\prime}$, which gives us

$$
\begin{aligned}
\mathbf{N}= & \int_{0}^{D} \tilde{\mathbf{J}}\left(z^{\prime}, \omega\right) e^{i k z^{\prime} \cos \theta} d z^{\prime} \\
& \times \int_{0}^{R} \int_{0}^{2 \pi} e^{i k r_{\perp}^{\prime} \sin \theta \cos \phi^{\prime}} r_{\perp}^{\prime} d \phi^{\prime} d r_{\perp}^{\prime} \\
= & \frac{2 A J_{1}(k R \sin \theta)}{k R \sin \theta} \int_{0}^{D} \tilde{\mathbf{J}}\left(z^{\prime}, \omega\right) e^{i k z^{\prime} \cos \theta} d z^{\prime},
\end{aligned}
$$

where $D$ is the thickness of the DSM, $A=\pi R^{2}$ is the area of the circular radiating region (we choose $D=250 \mathrm{~nm}$ and $R=1 \mathrm{~mm}$ throughout our work), and $J_{1}$ is the Bessel function of the first order. Substituting $\tilde{\mathbf{A}}(\mathbf{r}, \omega)$ into the Lorenz gauge field expression

$$
\tilde{\mathbf{E}}(\mathbf{r}, \omega)=-i \omega \tilde{\mathbf{A}}(\mathbf{r}, \omega)-\frac{i \omega}{k^{2}} \nabla[\nabla \cdot \tilde{\mathbf{A}}(\mathbf{r}, \omega)]
$$

and retaining the terms that fall no faster than $1 / r$, we find the angular field components:

$$
\begin{aligned}
\left\{\begin{array}{c}
\tilde{E}_{\theta}(\mathbf{r}, \omega) \\
\tilde{E}_{\phi}(\mathbf{r}, \omega)
\end{array}\right\} & =\left\{\begin{array}{c}
\eta_{0} \tilde{H}_{\phi}(\mathbf{r}, \omega) \\
-\eta_{0} \tilde{H}_{\theta}(\mathbf{r}, \omega)
\end{array}\right\} \\
& =-\frac{i k \eta_{0} e^{-i k r}}{4 \pi r}\left\{\begin{array}{l}
N_{\theta} \\
N_{\phi}
\end{array}\right\},
\end{aligned}
$$

where $\eta_{0}=\mu_{0} c$ is the vacuum impedance. Considering the time-domain fields

$$
E_{\phi, \theta}(\mathbf{r}, t)=\frac{1}{2 \pi} \int \tilde{E}_{\phi, \theta}(\mathbf{r}, \omega) e^{i \omega t} d \omega
$$

and setting $\mathbf{J} \| \hat{\mathbf{x}}$, the radial component of the Poynting vector reads

$$
\begin{aligned}
\frac{d^{2} U}{d t d A} & =\frac{1}{2 \eta_{0}}\left\{\left|E_{\theta}(\mathbf{r}, t)\right|^{2}+\left|E_{\phi}(\mathbf{r}, t)\right|^{2}\right\} \\
& =\frac{A^{2}}{8 \pi^{2} r^{2} \epsilon_{0} c}|F(\theta, t)|^{2}\left(\cos ^{2} \phi \cos ^{2} \theta+\sin ^{2} \phi\right),
\end{aligned}
$$

where

$$
\begin{aligned}
F(\theta, t)= & \frac{1}{2 \pi} \int\left[i k e^{-i k r+i \omega t} \frac{J_{1}(k R \sin \theta)}{k R \sin \theta}\right. \\
& \left.\times \int_{0}^{D} \tilde{J}_{x}\left(z^{\prime}, \omega\right) e^{i k z^{\prime} \cos \theta} d z^{\prime}\right] d \omega .
\end{aligned}
$$

Using Parseval's theorem, we find that the energy radiated per unit solid angle $\Omega$ and unit angular frequency in the far field is given by

$$
\frac{d^{2} U}{d \omega d \Omega}=\frac{A^{2}}{8 \pi^{3} \epsilon_{0} c}\left(\cos ^{2} \phi \cos ^{2} \theta+\sin ^{2} \phi\right)|\tilde{F}(\theta, \omega)|^{2},
$$

where $\tilde{F}(\theta, \omega)$ is the Fourier transform of $F(\theta, t)$. For the 2D DSMs, we consider a volume current density given by $\mathbf{J}=\mathbf{K}_{s} \delta(z)$, where $\mathbf{K}_{s}$ is the surface current induced in the 2D DSM sheet centered at $z=0$.

For Fig. 1 in the main text, we numerically evaluate the integral over $z^{\prime}$ in Eq. (A35) based on our FDTD results, followed by numerically integrating the intensity spectrum, given by Eq. (A36), over all solid angles in the forward emission direction to obtain the energy spectral density $d U / d \omega$. To produce the spectrum in units of photons per $1 \%$ bandwidth (BW), we divide $d U / d \omega$ by a factor $100 \hbar$. For Figs. 2 and 3 , we present our results using Eq. (A36) evaluated at $\theta=0$ (forward emission) and assume that the current is spatially uniform throughout the entire sample volume.

\section{Computing the energy conversion efficiency of HHG}

The energy conversion efficiency is defined as the ratio of the energy of the $N$ th harmonic, $U_{N}$, to the incident pulse energy, $U_{\text {in }}$. The incident pulse energy is computed as

$$
U_{\text {in }}=\frac{A}{2 \mu_{0} c} \int\left|E_{\mathrm{in}, x}(t)\right|^{2} d t,
$$

where $E_{\mathrm{in}, x}(t)$ is the temporal profile of the incident driving electric field and $A$ is the area of the sample as defined in Eq. (A36). To obtain the energy conversion efficiency of the $N$ th harmonic, we integrate the energy spectral density $d U / d \omega$ over the frequency domain from $(N-1) \omega_{0}$ to $(N+$ 1) $\omega_{0}$.

\section{APPENDIX B: DERIVATION OF CLOSED FORM, ANALYTICAL EXPRESSIONS FOR INTRABAND CURRENT}

Here, we derive nonperturbative expressions for the intraband current induced in 3D and 2D Dirac semimetals (DSMs). Unless otherwise stated, all notation used is as defined in the 
main text. We begin by considering Eq. (1) in the main text. When the driving frequencies $\omega$ satisfy the relation $\hbar \omega \ll$ $2 \mathcal{E}_{\mathrm{F}}$, the intraband contribution dominates. Under these conditions, we are justified in setting $\Gamma_{\mathbf{p}}(t)=0$ and $\mathcal{N}_{\mathbf{p}}(t)=\mathcal{N}_{\mathbf{p}}\left(t_{0}\right)$ at all times, effectively neglecting interband transitions. Here, $\mathcal{N}_{\mathbf{p}}\left(t_{0}\right)=f_{\mathrm{D}}\left[\mathcal{E}\left(t_{0}\right)\right]-f_{\mathrm{D}}\left[-\mathcal{E}\left(t_{0}\right)\right]$ is the initial population inversion, where $f_{\mathrm{D}}(\mathcal{E})=\left\{1+\exp \left[(\mathcal{E}-\mu(T)) / k_{\mathrm{B}} T\right]\right\}^{-1}$ is the Fermi-Dirac distribution, $\mathcal{E}\left(t_{0}\right)=\sqrt{\sum_{i} v_{i}^{2} p_{i}^{2}}$ is the initial energy in the absence of any fields, $v_{i}$ are the Fermi velocities, $p_{i}$ are the initial electron momenta, $i \in\{x, y, z\}(i \in\{x, y\})$ is the Cartesian coordinate for a 3D DSM (2D DSM), $\mu(T)$ is the chemical potential at temperature $T$, and $k_{\mathrm{B}}$ is the Boltzmann constant. In the limit $T \rightarrow 0 \mathrm{~K}$ and in the absence of scattering $(\tau \rightarrow \infty)$, Eq. (1) in the main text becomes

$$
J_{i}=\frac{g e v_{i}}{(2 \pi \hbar)^{n}} \int\left[1-\Theta\left(|\mathbf{p}|-p_{\mathrm{F}}\right)\right] \frac{v_{i} \pi_{i}(t)}{\mathcal{E}(t)} d^{n} \mathbf{p},
$$

where $\Theta$ is the Heaviside step function, $p_{\mathrm{F}}$ is the Fermi momentum, $\pi_{i}=p_{i}+e A_{i}$ is the minimal-coupling momentum component along direction $i$, and $\mathcal{E}(t)=\sqrt{\sum_{j} v_{j}^{2} \pi_{j}^{2}(t)}$ is the instantaneous energy. We set $n=3(n=2)$ for 3D (2D) DSMs. For convenience, we perform a change of variables $\mathbf{q}=\left(v_{x} p_{x}, v_{y} p_{y}, v_{z} p_{z}\right)$, which allows us to recast the above integral as

$$
J_{i} \propto \int\left[1-\Theta\left(|\mathbf{q}|-\mathcal{E}_{\mathrm{F}}\right)\right] \frac{q_{i}+e v_{i} A_{i}(t)}{\mathcal{E}(t)} d^{n} \mathbf{q} .
$$

Using this equation as a starting point, we derive separately the intraband current for the $3 \mathrm{D}$ case followed by the $2 \mathrm{D}$ case.

\section{3D Dirac semimetals}

Here, we derive a nonperturbative expression for the $x$ component of the intraband current. The procedure used here can be applied to obtain nonperturbative expressions for any component of the intraband current. Setting $i=x$, Eq. (B2) becomes

$$
J_{x}(t)=-\frac{g e}{(2 \pi \hbar)^{3} v_{y} v_{z}} \iiint \frac{q_{x}+\mathcal{A}_{x}}{\mathcal{E}(t)} d^{3} \mathbf{q},
$$

where we have introduced the scaled vector potential $\mathcal{A}_{i}=$ $e v_{i} A_{i}$, with $i \in\{x, y, z\}$. The integration region is a sphere in $\mathbf{q}$ space with radius $\mathcal{E}_{\mathrm{F}}$. Integrating over $q_{x}$, we obtain

$$
\begin{aligned}
J_{x}= & -\frac{g e}{8 \pi^{3} \hbar^{3} v_{y} v_{z}} \int_{-\mathcal{E}_{\mathrm{F}}}^{+\mathcal{E}_{\mathrm{F}}} d q_{z} \int_{-\sqrt{\mathcal{E}_{\mathrm{F}}^{2}-q_{z}^{2}}}^{+\sqrt{\mathcal{E}_{\mathrm{F}}^{2}-q_{z}^{2}}} d q_{y}\left\{\sqrt{\mathcal{E}_{\mathrm{F}}^{2}+|\mathcal{A}|^{2}+2 q_{y} \mathcal{A}_{y}+2 q_{z} \mathcal{A}_{z}+2 \mathcal{A}_{x} \sqrt{\mathcal{E}_{\mathrm{F}}^{2}-q_{y}^{2}-q_{z}^{2}}}\right. \\
& \left.-\sqrt{\mathcal{E}_{\mathrm{F}}^{2}+|\mathcal{A}|^{2}+2 q_{y} \mathcal{A}_{y}+2 q_{z} \mathcal{A}_{z}-2 \mathcal{A}_{x} \sqrt{\mathcal{E}_{\mathrm{F}}^{2}-q_{y}^{2}-q_{z}^{2}}}\right\}
\end{aligned}
$$

where $|\mathcal{A}|=\sqrt{\mathcal{A}_{x}^{2}+\mathcal{A}_{y}^{2}+\mathcal{A}_{z}^{2}}$. We use polar coordinates in the $q_{y}-q_{z}$ plane: $q_{y}=q_{\perp} \cos \varphi, q_{z}=q_{\perp} \sin \varphi, q_{\perp}^{2}=q_{y}^{2}+q_{z}^{2}$. The integral then becomes

$$
\begin{aligned}
J_{x} \propto & \int_{0}^{2 \pi} d \varphi \int_{0}^{\mathcal{E}_{\mathrm{F}}} d q_{\perp} q_{\perp}\left\{\sqrt{\mathcal{E}_{\mathrm{F}}^{2}+|\mathcal{A}|^{2}+2 q_{\perp}\left(\mathcal{A}_{y} \cos \varphi+\mathcal{A}_{z} \sin \varphi\right)+2 \mathcal{A}_{x} \sqrt{\mathcal{E}_{\mathrm{F}}^{2}-q_{\perp}^{2}}}\right. \\
& \left.-\sqrt{\mathcal{E}_{\mathrm{F}}^{2}+|\mathcal{A}|^{2}+2 q_{\perp}\left(\mathcal{A}_{y} \cos \varphi+\mathcal{A}_{z} \sin \varphi\right)-2 \mathcal{A}_{x} \sqrt{\mathcal{E}_{\mathrm{F}}^{2}-q_{\perp}^{2}}}\right\} .
\end{aligned}
$$

We now define $q_{\perp}=\mathcal{E}_{\mathrm{F}} \sin \Psi$, where $\Psi$ goes from 0 to $\pi / 2$ :

$$
\begin{aligned}
J_{x} \propto & \frac{\mathcal{E}_{\mathrm{F}}^{2}}{2} \int_{0}^{2 \pi} d \varphi \int_{0}^{\pi / 2} d \Psi \sin 2 \Psi\left\{\sqrt{\mathcal{E}_{\mathrm{F}}^{2}+|\mathcal{A}|^{2}+2 \mathcal{E}_{\mathrm{F}} \sin \Psi\left(\mathcal{A}_{y} \cos \varphi+\mathcal{A}_{z} \sin \varphi\right)+2 \mathcal{A}_{x} \mathcal{E}_{\mathrm{F}} \cos \Psi}\right. \\
& \left.-\sqrt{\mathcal{E}_{\mathrm{F}}^{2}+|\mathcal{A}|^{2}+2 \mathcal{E}_{\mathrm{F}} \sin \Psi\left(\mathcal{A}_{y} \cos \varphi+\mathcal{A}_{z} \sin \varphi\right)-2 \mathcal{A}_{x} \mathcal{E}_{\mathrm{F}} \cos \Psi}\right\} .
\end{aligned}
$$

We can express each of the square root terms as a series, and the integrand becomes

$$
\begin{aligned}
& \sin 2 \Psi \sqrt{\mathcal{E}_{\mathrm{F}}^{2}+|\mathcal{A}|^{2}} \sum_{n=0}^{\infty} \frac{\Gamma(3 / 2)}{\Gamma(n+1) \Gamma\left(\frac{3}{2}-n\right)}\left(\frac{2 \mathcal{E}_{\mathrm{F}}}{\mathcal{E}_{\mathrm{F}}^{2}+|\mathcal{A}|^{2}}\right)^{n}\left\{\left[\sin \Psi\left(\mathcal{A}_{y} \cos \varphi+\mathcal{A}_{z} \sin \varphi\right)+\mathcal{A}_{x} \cos \Psi\right]^{n}\right. \\
& \left.-\left[\sin \Psi\left(\mathcal{A}_{y} \cos \varphi+\mathcal{A}_{z} \sin \varphi\right)-\mathcal{A}_{x} \cos \Psi\right]^{n}\right\} .
\end{aligned}
$$

From the binomial theorem, we have

$$
(a+b)^{n}-(a-b)^{n}=\sum_{l=0}^{n} \frac{\Gamma(n+1)}{\Gamma(l+1) \Gamma(n-l+1)}\left[a^{n-l} b^{l}-a^{n-l}(-b)^{l}\right]=\sum_{l \in \mathbb{Z}^{+, \text {odd }}}^{n} \frac{\Gamma(n+1)}{\Gamma(l+1) \Gamma(n-l+1)} 2 a^{n-l} b^{l}
$$


We can use this identity by making the substitutions $a=\sin \Psi\left(\mathcal{A}_{y} \cos \varphi+\mathcal{A}_{z} \sin \varphi\right)$ and $b=\mathcal{A}_{x} \cos \Psi$. So, Eq. (B7) can be written as

$$
\begin{aligned}
\sin 2 & \Psi \sqrt{\mathcal{E}_{\mathrm{F}}^{2}+|\mathcal{A}|^{2}}\left\{\sum _ { n = 0 } ^ { \infty } \frac { \Gamma ( 3 / 2 ) } { \Gamma ( n + 1 ) \Gamma ( \frac { 3 } { 2 } - n ) } ( \frac { 2 \mathcal { E } _ { \mathrm { F } } } { \mathcal { E } _ { \mathrm { F } } ^ { 2 } + | \mathcal { A } | ^ { 2 } } ) ^ { n } \left[\sum_{l \in \mathbb{Z}^{+, \text {odd }}}^{n} \frac{\Gamma(n+1)}{\Gamma(l+1) \Gamma(n-l+1)} 2 \sin ^{n-l} \Psi\right.\right. \\
& \left.\left.\times\left(\mathcal{A}_{y} \cos \varphi+\mathcal{A}_{z} \sin \varphi\right)^{n-l} \mathcal{A}_{x}^{l} \cos ^{l} \Psi\right]\right\} \\
= & 2 \sqrt{\mathcal{E}_{\mathrm{F}}^{2}+|\mathcal{A}|^{2}}\left\{\sum _ { n = 0 } ^ { \infty } \frac { \Gamma ( 3 / 2 ) } { \Gamma ( n + 1 ) \Gamma ( \frac { 3 } { 2 } - n ) } ( \frac { 2 \mathcal { E } _ { \mathrm { F } } } { \mathcal { E } _ { \mathrm { F } } ^ { 2 } + | \mathcal { A } | ^ { 2 } } ) ^ { n } \left[\sum_{l \in \mathbb{Z}^{+, \text {odd }}}^{n} \frac{\Gamma(n+1)}{\Gamma(l+1) \Gamma(n-l+1)} 2 \sin ^{n-l+1} \Psi\right.\right. \\
& \left.\left.\times \cos ^{l+1} \Psi\left(\mathcal{A}_{y} \cos \varphi+\mathcal{A}_{z} \sin \varphi\right)^{n-l} \mathcal{A}_{x}^{l}\right]\right\} .
\end{aligned}
$$

Now that we have untangled the terms that depend on $\Psi$ and $\varphi$, we can bring the integrals into the innermost summation. For the integral over $\Psi$, we find

$$
\int_{0}^{\pi / 2} \sin ^{n-l+1} \Psi \cos ^{l+1} \Psi d \Psi=\frac{\Gamma\left(\frac{l+2}{2}\right) \Gamma\left(\frac{n-l+2}{2}\right)}{2 \Gamma\left(2+\frac{n}{2}\right)}
$$

For the integral over $\varphi$, we have

$$
\int_{0}^{2 \pi}\left(\mathcal{A}_{y} \cos \varphi+\mathcal{A}_{z} \sin \varphi\right)^{n-l} d \varphi=\frac{\sqrt{\pi} \Gamma\left(\frac{1+n-l}{2}\right)}{\Gamma\left(\frac{2+n-l}{2}\right)}\left[1+(-1)^{n-l}\right]\left(\mathcal{A}_{y}^{2}+\mathcal{A}_{z}^{2}\right)^{\frac{n-l}{2}} .
$$

Note that if $\mathcal{A}_{y}=\mathcal{A}_{z}=0$, only the $l=n$ term in the inner summation exists; the $l=1,3,5, \ldots, n-2$ terms all vanish. This can be even further simplified: $1+(-1)^{n-l}$ will vanish if $n-l \in$ odd. Since $l \in$ odd $\Rightarrow$ if $n \in$ even, then $n-l \in$ odd. So the only nonvanishing contributions will occur when $n \in$ odd, which means $1+(-1)^{n-l}=2 \forall n, l \in$ odd. Therefore, the full result of the integral is

$$
J_{x}=-\frac{g e}{8 \pi^{2} \hbar^{3} v_{y} v_{z}}\left\{\sum_{n \in \mathbb{Z}^{+, \text {odd }}}^{\infty} \frac{1}{\Gamma\left(\frac{3}{2}-n\right)} \frac{2^{n} \mathcal{E}_{\mathrm{F}}^{n+2}}{\left(\mathcal{E}_{\mathrm{F}}^{2}+|\mathcal{A}|^{2}\right)^{n-1 / 2}}\left[\sum_{l \in \mathbb{Z}^{+, \text {odd }}}^{n} \frac{\Gamma\left(\frac{l+2}{2}\right) \Gamma\left(\frac{1+n-l}{2}\right)}{\Gamma(l+1) \Gamma(n-l+1) \Gamma\left(2+\frac{n}{2}\right)}\left|\mathcal{A}_{\perp}\right|^{n-l} \mathcal{A}_{x}^{l}\right]\right\},
$$

where $\left|\mathcal{A}_{\perp}\right|=\sqrt{\mathcal{A}_{y}^{2}+\mathcal{A}_{z}^{2}}$. For the most general case of a current in any $i \in\{x, y, z\}$ direction, the expression is

$$
J_{i}=\frac{-g e}{8 \pi^{2} \hbar^{3} v_{j} v_{k}}\left\{\sum_{n \in \mathbb{Z}^{+, \text {odd }}}^{\infty} \frac{1}{\Gamma\left(\frac{3}{2}-n\right)} \frac{2^{n} \mathcal{E}_{\mathrm{F}}^{n+2}}{\left(\mathcal{E}_{\mathrm{F}}^{2}+|\mathcal{A}|^{2}\right)^{n-1 / 2}}\left[\sum_{l \in \mathbb{Z}^{+, \text {odd }}}^{n} \frac{\Gamma\left(\frac{l+2}{2}\right) \Gamma\left(\frac{1+n-l}{2}\right)}{\Gamma(l+1) \Gamma(n-l+1) \Gamma\left(2+\frac{n}{2}\right)}\left|\mathcal{A}_{\perp}\right|^{n-l} \mathcal{A}_{i}^{l}\right]\right\}
$$

where $j, k \in\{x, y, z\}$ such that $i \neq j \neq k$, and $\left|\mathcal{A}_{\perp}\right|=\sqrt{\mathcal{A}_{j}^{2}+\mathcal{A}_{k}^{2}}$. We define the potential $\Phi(t) \equiv|\mathcal{A}| / e=\sqrt{\sum_{i} v_{i}^{2} A_{i}^{2}}$, where $i \in\{x, y, z\}$. When the combination of driving fields obeys $\Phi(t)<\Phi_{\text {crit }}$, where $\Phi_{\text {crit }} \equiv \mathcal{E}_{\mathrm{F}} / e$ is defined to be the critical potential, we can evaluate terms of arbitrary order in $A(t)$ to show that this complicated series expression only has contributions from terms with cumulative powers of 1 and 3 with respect to the vector potential (we have done this up to $n=31$, involving lengthy and tedious calculations):

$$
J_{i}^{3 \mathrm{D}, \mathrm{sub}}(t)=-\frac{g e^{2} v_{i}}{6 \pi^{2} \hbar^{3} v_{j} v_{k}} A_{i}(t)\left[\mathcal{E}_{\mathrm{F}}^{2}-\frac{e^{2}}{5} \sum_{l} v_{l}^{2} A_{l}^{2}(t)\right],
$$

where $l \in\{x, y, z\}$. Equation (B14) is exactly the same as Eq. (2) in the main text. The complete absence of higher order intraband components is further verified through rigorous numerical simulations of Eq. (1), which yield results in excellent agreement with Eq. (B14). Similarly, when $\Phi(t)>\Phi_{\text {crit }}$, we obtain the expression

$$
J_{i}^{3 \mathrm{D}, \text { sup }}(t)=-\frac{g e \mathcal{E}_{F}^{3}}{6 \pi^{2} \hbar^{3} v_{j} v_{k}} \frac{v_{i} A_{i}(t)}{\sqrt{\sum_{l} v_{l}^{2} A_{l}^{2}(t)}}\left[1-\frac{\mathcal{E}_{\mathrm{F}}^{2}}{5 e^{2} \sum_{l} v_{l}^{2} A_{l}^{2}(t)}\right],
$$

which is exactly Eq. (3) in the main text. It should also be noted that depending on the instantaneous value of $\Phi(t)$, the current expression is piecewise. For a large enough $\Phi_{\max }$, which is the maximum amplitude of $\Phi(t)$, the expression that describes the current switches between Eqs. (B14) and (B15), with both expressions being continuous at $\Phi(t)=\Phi_{\text {crit }}$. Here, we have adopted a brute-force, series approach in deriving Eqs. (B14) and (B15), thereby showing that all intraband nonlinearities beyond the third order vanish in the subcritical regime $\left(\Phi_{\max }<\Phi_{\text {crit }}\right)$ through Eq. (B14). In Appendix E, we consider a simplified case in which the 3D DSM is driven by $\mathbf{A}(t)=\left(A_{x}(t), 0,0\right)$, which reveals that the higher orders are suppressed due to both the 
presence of Legendre polynomials in the single-electron current and the spherical integration geometry in a scaled momentum space.

\section{The 2D Dirac semimetals}

We now consider the induced intraband current in a 2D DSM. Once again, we consider $J_{x}$, but the derivations are also applicable to the $y$ component of the current $\mathbf{J}$. We start from

$$
J_{x}(t)=-\frac{g e}{(2 \pi \hbar)^{2} v_{y}} \iint d^{2} \mathbf{q}\left[1-\Theta\left(|\mathbf{q}|-\mathcal{E}_{\mathrm{F}}\right)\right] \frac{q_{x}+\mathcal{A}_{x}}{\mathcal{E}(t)},
$$

where $\mathcal{E}(t)=\sqrt{\left(q_{x}+\mathcal{A}_{x}\right)^{2}+\left(q_{y}+\mathcal{A}_{y}\right)^{2}}$, and integrate over $q_{x}$ :

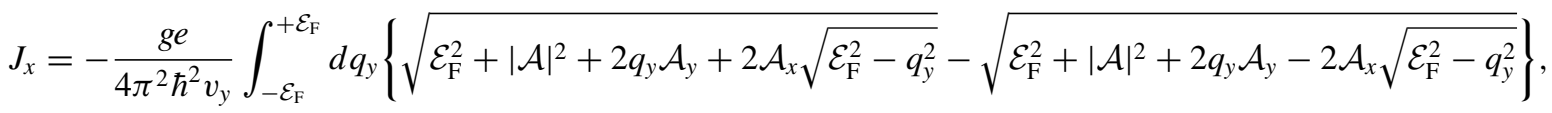

where $|\mathcal{A}|=\sqrt{\mathcal{A}_{x}^{2}+\mathcal{A}_{y}^{2}}$. Performing the change of variables $q_{y}=\mathcal{E}_{\mathrm{F}} \sin \varphi$, where $\varphi \in[-\pi / 2,+\pi / 2]$, we find

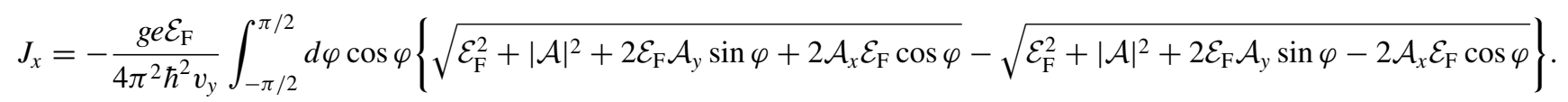

Once again expressing the square-root terms as a series expression, we obtain

$$
\begin{aligned}
J_{x}=- & \frac{g e \mathcal{E}_{\mathrm{F}}}{4 \pi^{2} \hbar^{2} v_{y}} \sqrt{\mathcal{E}_{\mathrm{F}}^{2}+|\mathcal{A}|^{2}} \sum_{n \in \mathbb{Z}^{+, \text {odd }}}^{\infty} \frac{\Gamma(3 / 2)}{\Gamma\left(\frac{3}{2}-n\right)}\left(\frac{2 \mathcal{E}_{\mathrm{F}}}{\mathcal{E}_{\mathrm{F}}^{2}+|\mathcal{A}|^{2}}\right)^{n} \\
& \times\left\{\sum_{l \in \mathbb{Z}^{+, \text {odd }}}^{n} \frac{2 \mathcal{A}_{y}^{n-l} A_{x}^{l}}{\Gamma(l+1) \Gamma(n-l+1)} \int_{-\pi / 2}^{\pi / 2} \sin ^{n-l} \varphi \cos ^{l+1} \varphi d \varphi\right\} .
\end{aligned}
$$

Noting that

$$
\int_{-\pi / 2}^{\pi / 2} \sin ^{n-l} \varphi \cos ^{l+1} \varphi d \varphi=\frac{\Gamma\left(1+\frac{l}{2}\right) \Gamma\left(\frac{1-l+n}{2}\right)}{\Gamma\left(\frac{3+n}{2}\right)}
$$

when $n, l \in \mathbb{Z}^{+, \text {odd }}$, the entire integral becomes

$$
J_{x}=-\frac{g e \mathcal{E}_{\mathrm{F}}}{4 \pi^{2} \hbar^{2} v_{y}} \sqrt{\mathcal{E}_{\mathrm{F}}^{2}+|\mathcal{A}|^{2}} \sum_{n \in \mathbb{Z}^{+, \text {odd }}}^{\infty} \frac{\sqrt{\pi}}{\Gamma\left(\frac{3}{2}-n\right) \Gamma\left(\frac{3+n}{2}\right)}\left(\frac{2 \mathcal{E}_{\mathrm{F}}}{\mathcal{E}_{\mathrm{F}}^{2}+|\mathcal{A}|^{2}}\right)^{n}\left\{\sum_{l \in \mathbb{Z}_{\text {odd }}}^{n} \frac{\Gamma\left(1+\frac{l}{2}\right) \Gamma\left(\frac{1-l+n}{2}\right)}{\Gamma(l+1) \Gamma(n-l+1)} \mathcal{A}_{y}^{n-l} A_{x}^{l}\right\} .
$$

When the potential $\Phi(t) \equiv|\mathcal{A}| / e=\sqrt{\sum_{i} v_{i}^{2} A_{i}^{2}(t)}$ satisfies $\Phi(t)<\Phi_{\text {crit }}$, where $i \in\{x, y\}$ and $\Phi_{\text {crit }} \equiv \mathcal{E}_{\mathrm{F}} / e$, the closed-form intraband current along the direction $i$ becomes

$$
\begin{aligned}
J_{i}^{2 \mathrm{D}, \mathrm{sub}}(t)=- & \frac{g e^{2} \mathcal{E}_{\mathrm{F}}}{4 \pi \hbar^{2} v_{j}} v_{i} A_{i}(t)+\frac{g e^{4}}{32 \pi \hbar^{2} v_{j} \mathcal{E}_{\mathrm{F}}} v_{i} A_{i}(t) \sum_{k} v_{k}^{2} A_{k}^{2}(t)+\frac{g e^{6}}{256 \pi \hbar^{2} v_{j} \mathcal{E}_{\mathrm{F}}^{3}} v_{i} A_{i}(t)\left[\sum_{k} v_{k}^{2} A_{k}^{2}(t)\right]^{2} \\
& +\frac{5 g e^{8}}{4096 \pi \hbar^{2} v_{j} \mathcal{E}_{\mathrm{F}}^{5}} v_{i} A_{i}(t)\left[\sum_{k} v_{k}^{2} A_{k}^{2}(t)\right]^{3}+\cdots,
\end{aligned}
$$

where $j \in\{x, y\}$ is such that $i \neq j, k \in\{x, y\}$, and the ellipses refer to higher order terms. When $\Phi(t)>\Phi$ crit, we find

$$
J_{i}^{2 \mathrm{D}, \sup }(t)=-\frac{g e \mathcal{E}_{\mathrm{F}}^{2}}{4 \pi \hbar^{2} v_{j}} \frac{v_{i} A_{i}(t)}{\sqrt{\sum_{k} v_{k}^{2} A_{k}^{2}(t)}}+\frac{g \mathcal{E}_{\mathrm{F}}^{4}}{32 \pi \hbar^{2} e v_{j}} \frac{v_{i} A_{i}(t)}{{\sqrt{\sum_{k} v_{k}^{2} A_{k}^{2}(t)}}^{3}}+\frac{g \mathcal{E}_{\mathrm{F}}^{6}}{256 \pi \hbar^{2} e^{3} v_{j}} \frac{v_{i} A_{i}(t)}{{\sqrt{\sum_{k} v_{k}^{2} A_{k}^{2}(t)}}^{5}}+\cdots
$$

In the limit in which $\mathrm{A}(t)=\left[A_{x}(t), 0\right]$, Eq. (B22) reduces to

$$
J_{x}(t)=-\frac{g e^{2} v_{x} \mathcal{E}_{\mathrm{F}}}{4 \pi \hbar^{2} v_{y}} A_{x}(t)+\frac{g e^{4} v_{x}^{3}}{32 \pi \hbar^{2} v_{y} \mathcal{E}_{\mathrm{F}}} A_{x}(t)^{3}+\frac{g e^{6} v_{x}^{5}}{256 \pi \hbar^{2} v_{y} \mathcal{E}_{\mathrm{F}}^{3}} A_{x}(t)^{5}+\frac{5 g e^{8} v_{x}^{7}}{4096 \pi \hbar^{2} v_{y} \mathcal{E}_{\mathrm{F}}^{5}} A_{x}(t)^{7}+\cdots,
$$




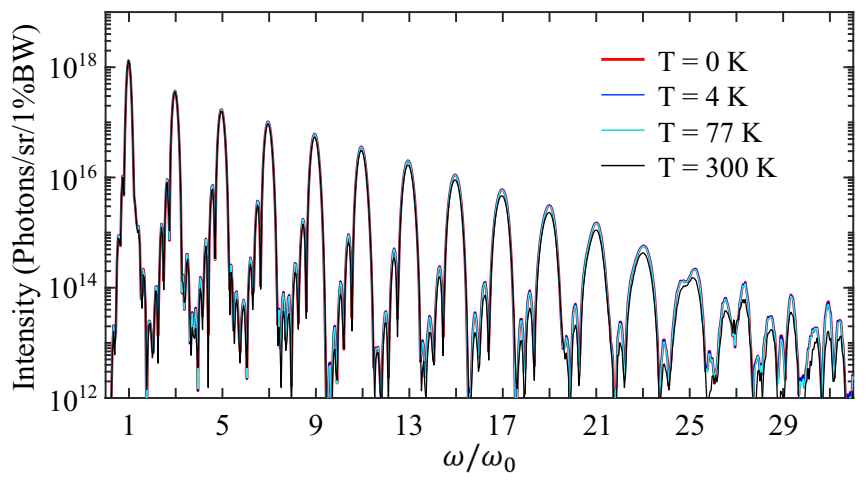

FIG. 6. HHG intensity spectrum in the supercritical regime at different temperatures. While the harmonic intensity peaks decrease as the temperature increases from $T=0 \mathrm{~K}$ to $T=300 \mathrm{~K}$, the changes are not substantial in the supercritical regime. We consider a $\mathrm{Cd}_{3} \mathrm{As}_{2}$ thin film doped to $\mathcal{E}_{\mathrm{F}}=250 \mathrm{meV}$ (at $T=0 \mathrm{~K}$ ) driven by a 2-ps-long, 1-THz-centered pulse of peak field strength $E_{x, 0}=10$ $\mathrm{MV} / \mathrm{m}$ inside the material. We assume that the induced current is spatially uniform throughout the 250-nm-thick thin film of radius $1 \mathrm{~mm}$. We neglect the effects of scattering.

which reproduces the result derived in Ref. [44] when $v_{x}=$ $v_{y}=v_{\mathrm{F}}$. Under the same conditions, but when $A_{x} \rightarrow \infty$, Eq. (B23) converges toward a square wave profile:

$$
\lim _{\left|A_{x}\right| \rightarrow \infty} J_{x}(t)=-\operatorname{sgn}\left[A_{x}(t)\right] \frac{g e \mathcal{E}_{\mathrm{F}}^{2}}{4 \pi \hbar^{2} v_{y}} .
$$

When the dispersion is isotropic and $g=4$ (spin and valley degeneracies), Eq. (B25) reduces to the intraband saturation current expression derived in Ref. [44]: $-\operatorname{sgn}\left[A_{x}(t)\right] e k_{\mathrm{F}} v_{\mathrm{F}} / \pi$.

\section{APPENDIX C: EFFECT OF TEMPERATURE AND CARRIER SCATTERING ON THE SUPERCRITICAL REGIME OF 3D DSMs}

In this section, we show the dependence of the HHG spectra of $\mathrm{Cd}_{3} \mathrm{As}_{2}$ in the supercritical regime when the effects of finite temperature and scattering times are included. As we see in Fig. 6, which include both intraband and interband contributions, the harmonic intensity peaks do not change significantly as a function of temperature. Figure 7 shows the dependence of HHG on scattering time, where we study a wide range of scattering times that includes measured values of $\approx 150 \mathrm{fs}$ [31]. Although the HHG response tends to deteriorate at shorter scattering times, it is interesting to note that this can be ameliorated by adjusting the Fermi level accordingly. Furthermore, the highly nonmonotonic dependence of HHG intensity on scattering time suggests that the scattering time, if it can be engineered, is a potential means of optimizing and controlling the relative intensities of different HHG peaks, and hence the spectral content of the output.

Figure 7 shows that the intraband harmonic intensity saturates as the scattering time increases. While a previous study [31] numerically predicted the same saturation trend for third-order harmonic generation in $\mathrm{Cd}_{3} \mathrm{As}_{2}$, we show that this same trend holds for harmonics of arbitrarily high orders, and present fully closed-form, analytical expressions [namely, Eqs. (2) and (3)] that capture this trend. Additionally, we

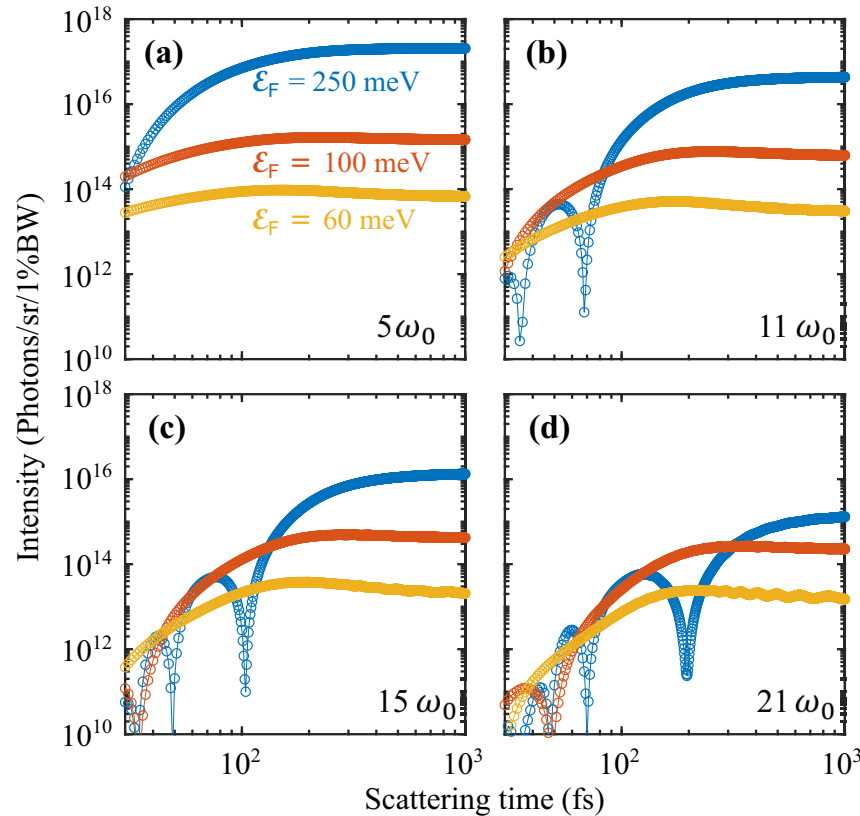

FIG. 7. Dependence of the HHG peak intensity on scattering time and Fermi level $\mathcal{E}_{\mathrm{F}}$. For all harmonics (frequency labeled in the bottom right corner of each panel), we observe that the peak intensities saturate as the scattering time increases. We find that in the supercritical regime, saturation occurs at a smaller scattering time for lower doping levels. As temperature has little effect on the HHG performance of $\mathrm{Cd}_{3} \mathrm{As}_{2}$ in the supercritical regime, we work in the $T \rightarrow 0 \mathrm{~K}$ limit. The values presented here are computed using Eqs. (B14) and (B15), with the vector potential modified to include intraband scattering. Unless otherwise stated, we consider the same parameters as in Fig. 6.

find that saturation occurs at faster scattering times when the doping level is lower. For instance, the 21st harmonic intensity [Fig. 7(d)] saturates at a scattering time of about $100 \mathrm{fs}$ for the samples doped to $\mathcal{E}_{\mathrm{F}}=100 \mathrm{meV}$ and $\mathcal{E}_{\mathrm{F}}=60 \mathrm{meV}$, but even at a scattering time of $1 \mathrm{ps}$, the sample doped to $\mathcal{E}_{\mathrm{F}}=250 \mathrm{meV}$ has yet to achieve saturation. The fact that saturation occurs at faster scattering times for DSMs doped to lower Fermi levels can be explained as follows: For a lower value of $\mathcal{E}_{\mathrm{F}}$, the Fermi surface lies closer to the Dirac point; hence, for a fixed driving field amplitude, a shorter time is required to accelerate the electron from the Fermi surface past the Dirac point; when the time taken to accelerate the electron past the Dirac point is significantly shorter than the scattering time, saturation occurs [31]. Thus, operating deeper in the supercritical regime (either by lowering $\mathcal{E}_{\mathrm{F}}$ or increasing the peak field amplitude, or both) is beneficial in limiting losses from scattering, allowing the HHG performance to approach the no-scattering limit.

Figure 8 shows that even when realistic scattering values are considered, HHG conversion efficiencies similar to the noscattering limit [Fig. 1(b)] can still be achieved. We see that for a scattering time of $150 \mathrm{fs}$ (black curves and filled circles), which is similar to experimentally determined values [31]; both the energy spectral density (left vertical axis) and energy conversion efficiencies (right vertical axis) of all harmonics up to the 31 st order remain within one order of magnitude of 


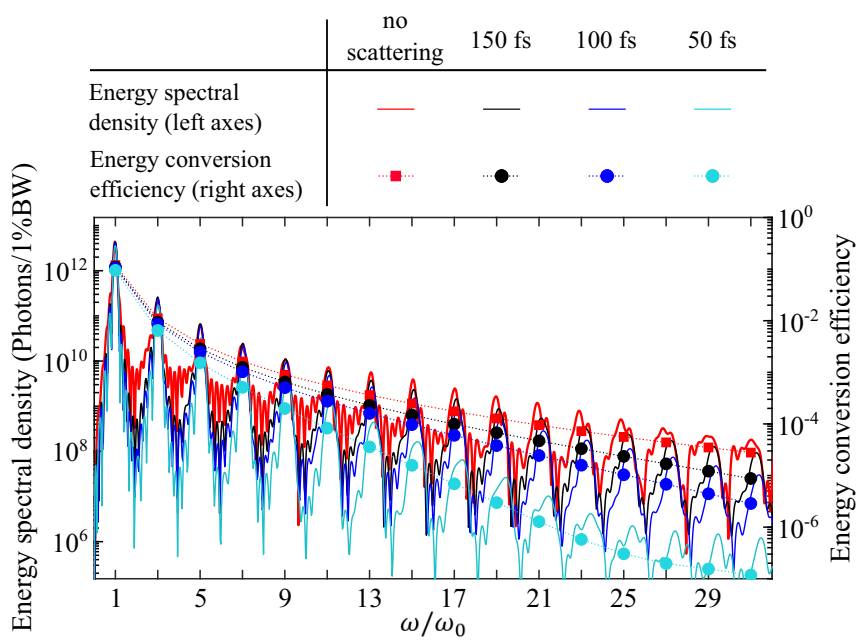

FIG. 8. High-order harmonic spectrum of $\mathrm{Cd}_{3} \mathrm{As}_{2}$ for various scattering times. The red curves and squares represent the situation in which scattering is neglected, which we plot in Fig. 1(b) in the main text. For a scattering time of 150 fs (black curves and filled circles), which is similar to the experimentally determined value of 145 fs obtained in Ref. [31], we see that the energy spectral density (left vertical axis) remains within the same order of magnitude as in the absence of scattering. The energy conversion efficiency (right vertical axis) of the 31 st harmonic is about $8.7 \times 10^{-6}$-within one order of magnitude of the case with no scattering. The decrease in both the energy spectral density and energy conversion efficiency is more dramatic for scattering times of the order of tens of fs, but the generation of harmonics up to the 31 st order and beyond remains possible. Unless otherwise stated, the same parameters as in Fig. 1(b) are considered. the no-scattering case (red curves and filled squares). While faster scattering times of 100 fs (blue curves and filled circles) and $50 \mathrm{fs}$ (cyan curves and filled circles) result in a greater drop in HHG peak energy spectral densities and conversion efficiencies, we find that generating harmonics beyond the 31 st order is still possible.

\section{APPENDIX D: POLARIZATION DEPENDENCE OF INTRABAND EMISSION IN 2D AND 3D DSMs}

In this Appendix, we illustrate how the polarization of the driving fields coupled with the degree of Fermi velocity anisotropy affect the HHG efficiency of Dirac materials (both 2D and 3D). Figures 9(a)-9(c), which are obtained for $\mathrm{A}(t)=\left[A_{x}(t), A_{y}(t), 0\right]$, show that no intraband harmonics above third order can be generated in the subcritical regime, regardless of laser polarization and phase, and also independently of the anisotropy of the Fermi velocities (described by the ratio $\left.v_{y} / v_{x}\right)$. This suppression is in contrast to the behavior of 2D DSMs [Fig. 9(d)], where higher harmonics above third order can be generated. Figures $9(\mathrm{e})-9(\mathrm{~h})$, which depict the intraband harmonics in 2D and 3D DSMs within the supercritical regime, emphasize the strong impact of drivingfield polarization on the increase in relative HHG intensity of higher harmonics. We find that linearly polarized (LP) driving pulses are favorable for efficient harmonic generation in contrast to circularly polarized (CP) pulses. Additionally, our results show that for 3D DSMs, a larger Fermi velocity anisotropy leads to more efficient HHG when driven by $\mathrm{CP}$ pulses.

On the other extreme, when a 3D DSM with isotropic Fermi velocities is driven by a CP pulse of arbitrarily large field amplitude, no high-order harmonics are produced. This

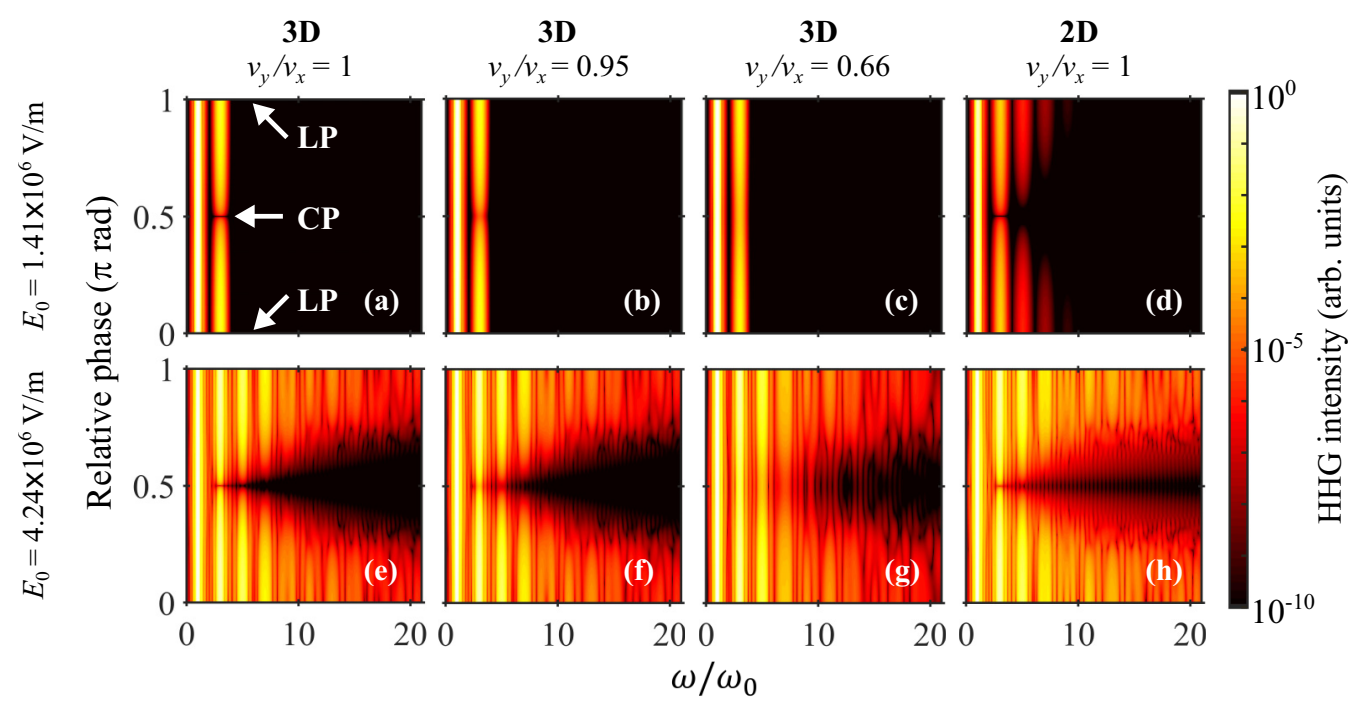

FIG. 9. Impact of polarization and Fermi velocity anisotropy on HHG in DSMs. The laser polarization is controlled by varying the relative phase between a superposed $x$-polarized and $y$-polarized laser pulse of the same amplitude $E_{0} / \sqrt{2}$ (labeled on the left). We choose the driving field amplitudes $E_{0}=1.41$ and $E_{0}=4.24 \mathrm{MV} / \mathrm{m}$, which correspond to the subcritical and supercritical regimes, respectively. In the subcritical regime of 3D DSMs, the strong suppression of HHG beyond the third harmonic persists for all polarizations (a)-(c), in contrast to 2D DSMs, where the fifth and ninth harmonics are relatively strong (d). In the supercritical regime (e)-(h), both 2D and 3D DSMs produce relatively high intensities of higher harmonics. Efficient HHG is most favored by linearly polarized (as opposed to circularly polarized) driving pulses in both 2D and 3D DSMs, a result that agrees with a previous study for graphene [67]. For 3D DSMs, we further observe that a larger Fermi velocity anisotropy generally leads to more effective HHG when driven by circularly polarized pulses. 
complete lack of intraband HHG can be explained by considering the case in which a DSM is driven by CP fields confined to the $x-y$ plane:

$$
\mathbf{A}(t)=A_{0} \cos \left(\omega_{0} t\right) \hat{\mathbf{x}}+A_{0} \sin \left(\omega_{0} t\right) \hat{\mathbf{y}},
$$

where $A_{0}=E_{0} / \omega_{0}$ is the amplitude of the vector potential and $E_{0}$ is the peak electric field strength. Then, the term $\sum_{i} v_{i}^{2} A_{i}^{2}(t)$, where $i \in\{x, y, z\}$, becomes

$$
\begin{aligned}
\sum_{i} v_{i}^{2} A_{i}^{2}(t) & =A_{0}^{2}\left[v_{x}^{2} \cos ^{2}\left(\omega_{0} t\right)+v_{y}^{2} \sin ^{2}\left(\omega_{0} t\right)\right] \\
& =\frac{A_{0}^{2}}{2}\left[\left(v_{x}^{2}+v_{y}^{2}\right)+\left(v_{x}^{2}-v_{y}^{2}\right) \cos \left(2 \omega_{0} t\right)\right] .
\end{aligned}
$$

This expression applies to both the 2D and 3D scenarios. We see that the suppression of the second term in the right-hand side scales as $\left(v_{x}^{2}-v_{y}^{2}\right)$. In the subcritical regime [Eqs. (B14) and (B22)], this term is responsible for the generation of higher harmonics, which is why 2D and 3D DSMs with $v_{x}=$ $v_{y}$ emit only the fundamental harmonic when they are driven by a CP pulse. It should be noted that while frequency multiplication is nonexistent, subcritical nonlinearities, which only serve to enhance the amplitude of the intraband current, still arise under these conditions due to the first term in Eq. (D2).

In the supercritical regime [Eqs. ((B15) and (B23)], we find that when $v_{x}=v_{y}$, a similar suppression of HHG occurs when driven by $\mathrm{CP}$ pulses, leaving only the fundamental frequency. This holds true even in the limit of large $A_{0}$ : Instead of the square-wave time-domain current profile we expect deep in the supercritical regime $\left(\Phi_{\max } \gg \Phi_{\text {crit }}\right)$, the current profile remains purely harmonic instead since Eq. (D2) remains constant in time. Hence, only the fundamental harmonic survives. For 2D DSMs like graphene, the increasing suppression of HHG with increasing ellipticity of the driving fields has been previously studied [67]. However, the analysis that we present here is the first to show that this trend also holds for 3D DSMs.

In Fig. 9, we tailor the field polarization by varying the relative phase between a wave linearly polarized (LP) along $x$ and another along $y$. Each wave is a 1-THz pulse of 2-ps duration [intensity full width half maximum (FWHM)]. Both waves have equal amplitudes $E_{0} / \sqrt{2}$ (labelled on the left). The 2D and 3D DSMs are taken to have a Fermi energy $\mathcal{E}_{\mathrm{F}}=250 \mathrm{meV}$ at temperature $T=0 \mathrm{~K}$. For all cases, we fix $v_{x}=v_{z}=10^{6} \mathrm{~m} / \mathrm{s}$. The spectral color maps are computed using only our closed-form analytical intraband expression given by Eqs. (B14) and (B15) for the 3D DSM case and Eqs. (B22) and (B23) for the 2D DSM case.

\section{APPENDIX E: ILLUSTRATING THE VANISHING OF HIGH-ORDER HARMONICS IN THE SUBCRITICAL REGIME WITH A LEGENDRE POLYNOMIAL EXPANSION}

In this Appendix, we show that the vanishing of all intraband nonlinearities beyond the third order arises geometrically from the additional dimension that 3D DSMs possess relative to $2 \mathrm{D}$ DSMs. In particular, we use a Legendre polynomial expansion to mathematically illustrate how the harmonics beyond the third order vanish in the subcritical regime $\left(\Phi_{\max }<\Phi_{\text {crit }}\right)$ of 3D DSMs. We do this by considering the simplified case in which the driving vector potential is linearly polarized: $\mathbf{A}(t)=\left[A_{x}(t), 0,0\right]$. Starting from Eq. (B3), we obtain

$$
J_{x}(t)=-\frac{g e}{(2 \pi \hbar)^{3} v_{y} v_{z}} \iiint \frac{q_{x}+\mathcal{A}_{x}(t)}{\sqrt{\left[q_{x}+\mathcal{A}_{x}(t)\right]^{2}+q_{y}^{2}+q_{z}^{2}}} d^{3} \mathbf{q} .
$$

The integration region is a sphere of radius $\mathcal{E}_{\mathrm{F}}$ about the origin in scaled momentum space. We align the polar axis along the polarization direction $q_{x}$ and convert to spherical coordinates, allowing us to recast the integral as

$$
\begin{aligned}
J_{x}(t)= & -\frac{g e}{(2 \pi)^{2} \hbar^{3} v_{y} v_{z}} \\
& \times \iint \frac{q_{r} \cos \theta+\mathcal{A}_{x}}{\sqrt{q_{r}^{2}+\mathcal{A}_{x}^{2}+2 \mathcal{A}_{x} q_{r} \cos \theta}} q_{r}^{2} \sin \theta d \theta d q_{r},
\end{aligned}
$$

where we have integrated out a factor of $2 \pi$ over the azimuthal angle $\phi$. We first consider the case where $\mathcal{A}_{x}<0 \Rightarrow \mathcal{A}_{x}=$ $-\left|\mathcal{A}_{x}\right|$. Then, denoting $a_{x}=\left|\mathcal{A}_{x}\right|>0$ for compactness, our integral becomes

$$
\begin{aligned}
J_{x}= & -\frac{g e}{4 \pi^{2} \hbar^{3} v_{y} v_{z}} \\
& \times \int_{0}^{\mathcal{E}_{\mathrm{F}}} \int_{0}^{\pi} \frac{q_{r} \cos \theta-a_{x}}{\sqrt{q_{r}^{2}+a_{x}^{2}-2 a_{x} q_{r} \cos \theta}} q_{r}^{2} \sin \theta d \theta d q_{r} .
\end{aligned}
$$

When $a_{x}(t)$ satisfies $\Phi(t)<\Phi_{\text {crit }}$, we split the integral into two domains in $q_{r}: a_{x}<q_{r}$ and $a_{x}>q_{r}$. We define these as regions 1 and 2 respectively. The integrals for these regions are

$$
\begin{aligned}
& \mathcal{I}_{1}=\int_{a_{x}}^{\mathcal{E}_{\mathrm{F}}} \int_{0}^{\pi} \frac{q_{r} \cos \theta-a_{x}}{\sqrt{q_{r}^{2}+a_{x}^{2}-2 a_{x} q_{r} \cos \theta}} q_{r}^{2} \sin \theta d \theta d q_{r}, \\
& \mathcal{I}_{2}=\int_{0}^{a_{x}} \int_{0}^{\pi} \frac{q_{r} \cos \theta-a_{x}}{\sqrt{q_{r}^{2}+a_{x}^{2}-2 a_{x} q_{r} \cos \theta}} q_{r}^{2} \sin \theta d \theta d q_{r} .
\end{aligned}
$$

These integrals contain the generating function for the Legendre polynomials $P_{n}(\xi)$, where $\xi=\cos \theta$, and we express them in terms of that function as

$$
\begin{aligned}
& \mathcal{I}_{1}=\sum_{n=0}^{+\infty} \int_{a_{x}}^{\mathcal{E}_{\mathrm{F}}} \int_{-1}^{+1}\left[q_{r}^{2} \xi-a_{x} q_{r}\right] \frac{a_{x}^{n}}{q_{r}^{n}} P_{n}(\xi) d \xi d q_{r}, \\
& \mathcal{I}_{2}=\frac{1}{a_{x}} \sum_{n=0}^{+\infty} \int_{0}^{a_{x}} \int_{-1}^{+1}\left[q_{r}^{3} \xi-a_{x} q_{r}^{2}\right] \frac{q_{r}^{n}}{a_{x}^{n}} P_{n}(\xi) d \xi d q_{r} .
\end{aligned}
$$

In evaluating the above integrals, it is helpful to note that higher order Legendre polynomials vanish when integrated over all $\xi$ :

$$
\int_{-1}^{+1} P_{n}(\xi) d \xi=0, \forall n \geqslant 1
$$


Using $P_{0}(\xi)=1$ and $P_{1}(\xi)=\xi$, coupled with Bonnet's recursion formula

$$
(n+1) P_{n+1}(\xi)=(2 n+1) \xi P_{n}(\xi)-n P_{n-1}(\xi),
$$

we obtain a useful identity for $n \geqslant 2$ :

$$
\begin{aligned}
\int_{-1}^{+1} \xi P_{n}(\xi) d \xi \\
\quad=\int_{-1}^{+1} \frac{(n+1) P_{n+1}(\xi)+n P_{n-1}(\xi)}{2 n+1} d \xi=0 .
\end{aligned}
$$

In going to the last equality, we have used Eq. (E6). This implies that, for both regions, only $n=0$ and $n=1$ contribute:

$$
\begin{aligned}
& \mathcal{I}_{1}=\sum_{n=0}^{1} \int_{a_{x}}^{\mathcal{E}_{\mathrm{F}}} \int_{-1}^{+1} \frac{a_{x}^{n}}{q_{r}^{n-2}} \xi P_{n}(\xi)-\frac{a_{x}^{n+1}}{q_{r}^{n-1}} P_{n}(\xi) d \xi d q_{r}, \\
& \mathcal{I}_{2}=\frac{1}{a_{x}} \sum_{n=0}^{1} \int_{0}^{a_{x}} \int_{-1}^{+1} \frac{q_{r}^{n+3}}{a_{x}^{n}} \xi P_{n}(\xi)-\frac{q_{r}^{n+2}}{a_{x}^{n-1}} P_{n}(\xi) d \xi d q_{r} .
\end{aligned}
$$

Notice that $\xi$ is odd; this implies that, for both regions, only the $n=1$ and $n=0$ terms will not vanish upon integrating over $\xi$ for the first and second terms in the integrand, respectively. After integrating, we find

$$
\begin{aligned}
& \mathcal{I}_{1}=-\frac{2 \mathcal{E}_{\mathrm{F}}^{2}}{3} a_{x}+\frac{2}{3} a_{x}^{3}, \\
& \mathcal{I}_{2}=-\frac{8}{15} a_{x}^{3} .
\end{aligned}
$$

Summing both terms and restoring $a_{x}=\left|\mathcal{A}_{x}\right|=e v_{x}\left|A_{x}\right|$, we obtain

$$
J_{x}=-\frac{g e}{4 \pi^{2} \hbar^{3} v_{y} v_{z}}\left(-\frac{2 e v_{x} \mathcal{E}_{\mathrm{F}}^{2}}{3}\left|A_{x}\right|+\frac{2 e^{3} v_{x}^{3}}{15}\left|A_{x}\right|^{3}\right) .
$$

For $\mathcal{A}_{x}>0 \Rightarrow \mathcal{A}_{x}=\left|\mathcal{A}_{x}\right|$, we define $q_{x}=-q_{x}^{\prime}$. Solving the integral in the same way as above but defining $q_{x}^{\prime}=q_{r} \cos \theta$ instead, we can write

$$
J_{x}=-\frac{g e}{4 \pi^{2} \hbar^{3} v_{y} v_{z}}\left(\frac{2 e v_{x} \mathcal{E}_{\mathrm{F}}^{2}}{3}\left|A_{x}\right|-\frac{2 e^{3} v_{x}^{3}}{15}\left|A_{x}\right|^{3}\right) .
$$

Combining Eqs. (E11) and (E12), we obtain

$$
J_{x}(t)=-\sigma_{0}\left[\frac{2 g v_{x} \mathcal{E}_{\mathrm{F}}^{2}}{3 \pi^{2} \hbar^{2} v_{y} v_{z}} A_{x}(t)-\frac{2 g e^{2} v_{x}^{3}}{15 \pi^{2} \hbar^{2} v_{y} v_{z}} A_{x}(t)^{3}\right],
$$

where $\sigma_{0}=e^{2} / 4 \hbar$. For a general direction $i \in\{x, y, z\}$, and when $\Phi(t)<\Phi_{\text {crit }}$, the expression for the current becomes

$$
J_{i}^{3 \mathrm{D}, \mathrm{sub}}(t)=-\sigma_{0}\left[\frac{2 g v_{i} \mathcal{E}_{\mathrm{F}}^{2}}{3 \pi^{2} \hbar^{2} v_{j} v_{k}} A_{i}(t)-\frac{2 g e^{2} v_{i}^{3}}{15 \pi^{2} \hbar^{2} v_{j} v_{k}} A_{i}(t)^{3}\right],
$$

such that $i \neq j \neq k$. This shows that higher order terms vanish because (i) the single-electron intraband current at a specific momentum value can be expressed in terms of $P_{n}(\xi)$ and (ii) the integration geometry in $\mathbf{q}$ space is a sphere, which allows the integral to be recast into a form that cancels $P_{n}(\xi)$ for all $n \neq 0$ upon integration.

For completeness, we consider the case where $a_{x}(t)$ satisfies $\Phi(t)>\Phi_{\text {crit }}$, corresponding to the supercritical regime. Then, it suffices for us to solve for the region $a_{x}>\mathcal{E}_{\mathrm{F}}$ since our integral terminates at radial magnitude $\mathcal{E}_{\mathrm{F}}$ :

$$
\frac{1}{a_{x}} \sum_{n=0}^{+\infty} \int_{0}^{\mathcal{E}_{\mathrm{F}}} \int_{-1}^{+1}\left[q_{r}^{3} \xi-a_{x} q_{r}^{2}\right] \frac{q_{r}^{n}}{a_{x}^{n}} P_{n}(\xi) d \xi d q_{r} .
$$

Applying the same steps as the case where $\Phi(t)<\Phi_{\text {crit }}$, the intraband current expression for $\Phi(t)>\Phi_{\text {crit }}$ is

$$
\begin{aligned}
J_{i}^{3 \mathrm{D}, \sup }(t)= & -\operatorname{sgn}\left[A_{i}(t)\right] \\
& \times\left[\frac{g e \mathcal{E}_{\mathrm{F}}^{3}}{6 \pi^{2} \hbar^{3} v_{j} v_{k}}-\frac{g e \mathcal{E}_{\mathrm{F}}^{5}}{30 \pi^{2} \hbar^{3} e^{2} v_{i}^{2} v_{j} v_{k}} \frac{1}{A_{i}(t)^{2}}\right] .
\end{aligned}
$$

While Eq. (E16) also has only two terms instead of an infinite sum, the nonlinear response bears a greater resemblance to that of 2D DSMs. Deep in the supercritical regime $\left(\Phi_{\max } \gg\right.$ $\Phi_{\text {crit }}$ ), which in turn implies that $\left|A_{x}\right| \rightarrow \infty$, the intraband current converges toward a square-wave profile:

$$
\lim _{\left|A_{x}\right| \rightarrow \infty} J_{x}(t)=-\operatorname{sgn}\left[A_{x}(t)\right] \frac{g e \mathcal{E}_{\mathrm{F}}^{3}}{6 \pi^{2} \hbar^{3} v_{y} v_{z}} .
$$

This is reminiscent of the nonlinear response of a 2D DSM in the same limit [Eq. (B25)]. It should be noted that Eqs. (E14) and (E16) can be derived in a more straightforward manner via integration by substitution.

\section{APPENDIX F: EFFECT OF TEMPERATURE AND CARRIER SCATTERING ON THE SUBCRITICAL REGIME OF 3D DSMs}

As we have shown in Fig. 2 in the main text, in the limit where $T=0 \mathrm{~K}$ and in the absence of scattering, i.e., $\tau \rightarrow \infty$, 3D DSMs exhibit an anomalous suppression of HHG in the subcritical regime. In this section, we show that even when finite temperatures and scattering are included, the suppression of harmonics beyond the third order in $3 \mathrm{D} \mathrm{DSM} \mathrm{Cd} \mathrm{Cs}_{2}$ still persists.

Figures 10(a) and 10(b) show that for low but finite temperatures ( $T=4 \mathrm{~K}$ and $T=77 \mathrm{~K}$ respectively), the suppression of $\mathrm{HHG}$ is very strong, with the fifth-order harmonic and above at least $10^{7}$ times weaker compared to the third in all cases of Figs. 10(a) and 10(b). In fact, the spectra at both these temperatures are practically identical, indicating that this suppression can be observed without cryogenic temperatures. At $T=300 \mathrm{~K}$ (room temperature), Fig. 10(c) shows that the intensity of harmonics beyond the third order increases significantly due to the interband contribution: As the temperature increases, the Fermi-Dirac distribution gradually becomes smeared out about the Fermi level instead of a step function at low temperatures. As a result, at higher temperatures, more states in the conduction band are available for electrons to transition to, which implies an increase in interband current contribution to the higher harmonics. Nonetheless, the suppression of HHG in 3D DSMs still remains significant 


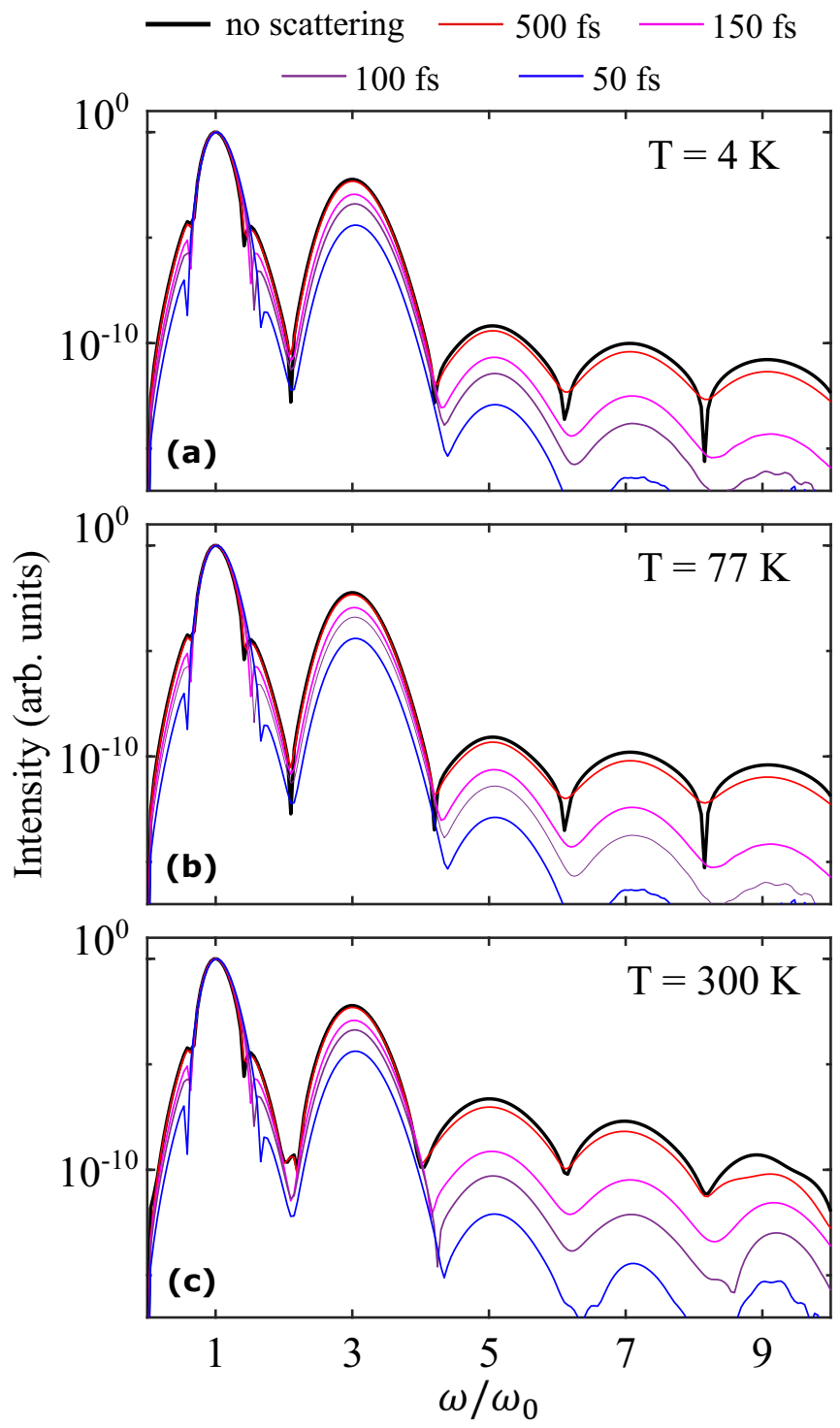

FIG. 10. Strong suppression of harmonics beyond third order from $\mathrm{Cd}_{3} \mathrm{As}_{2}$ in the subcritical regime at $T=4 \mathrm{~K}(\mathrm{a}), T=77 \mathrm{~K}$ (b), and $T=300 \mathrm{~K}$ (c) for various scattering times (different curves within each panel). While the suppression of HHG above third order is most obvious at $T=4 \mathrm{~K}$ and $T=77 \mathrm{~K}$, we see that this suppression still persists even up to room temperature $(T=300 \mathrm{~K})$. We also see that, at all temperatures, the inclusion of finite scattering only emphasizes the suppression. The spectra presented here are computed using Eq. (1), which accounts for both interband and intraband contributions. The driving field is a 2-ps-long laser pulse linearly polarized along $x$ with $1-\mathrm{THz}$ central frequency and peak amplitude $E_{x, 0}=1.1 \mathrm{MV} / \mathrm{m}$. The Fermi level is $\mathcal{E}_{\mathrm{F}}=250 \mathrm{meV}$. The temperature dependence of the chemical potential $\mu(T)$ is modeled using the Sommerfeld expansion, which is valid for highly doped samples [68].

even up to room temperature, with the fifth-order harmonic and above at least $10^{5}$ times weaker compared to the third in all cases of Fig. 10(c). Additionally, we note that inclusion of scattering only results in the suppression of HHG being more significant. Faster scattering times correlate with greater damping effects. We see that $\tau=500$ fs produces a spectrum

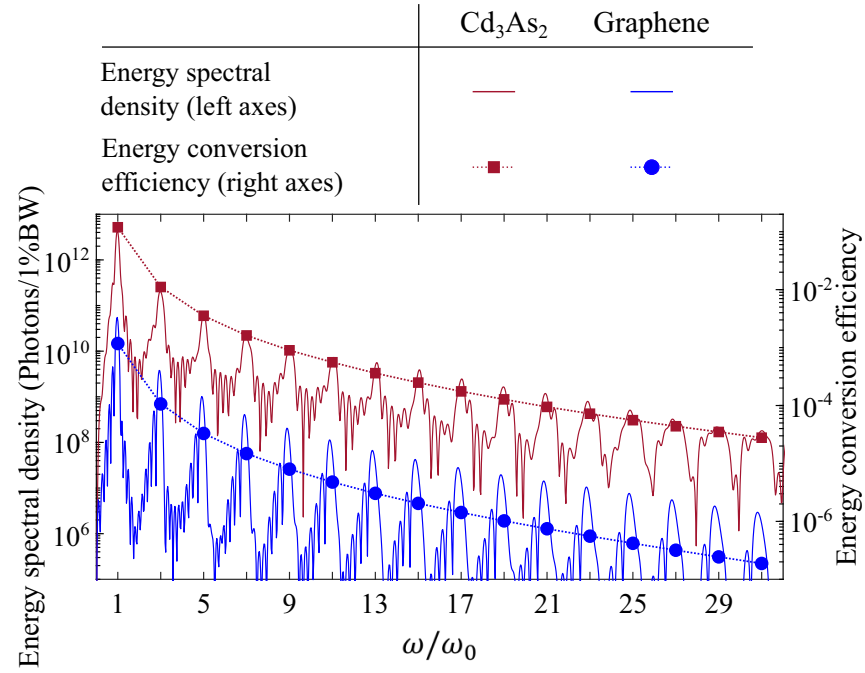

FIG. 11. Comparison of high-order harmonic generation in $\mathrm{Cd}_{3} \mathrm{As}_{2}$ (maroon curves, squares) and graphene (blue curves, circles). By virtue of a finite interaction volume, the intensity (left vertical axes) of the $\mathrm{HHG}$ in $\mathrm{Cd}_{3} \mathrm{As}_{2}$ can be more than two orders of magnitude greater than the HHG intensity output of a single atomic layer of graphene under the same conditions. For both materials, we assume a Fermi energy $\mathcal{E}_{\mathrm{F}}=60 \mathrm{meV}$ at $T=0$. We consider a cylindrical sample of radius $R=1 \mathrm{~mm}$ and thickness $D=250$ $\mathrm{nm}$ uniformly illuminated by a 1-THz-centered, 2-ps-long (intensity FWHM) linearly polarized (along $x$ ) laser pulse of peak amplitude $E_{x, 0}=10 \mathrm{MV} / \mathrm{m}$. These parameters and methodology are the same as those used in Fig. 1, with the FDTD algorithm used for graphene being replaced by a modified version detailed in Appendix A 5.

similar to the $\tau=\infty$ (no scattering) case, whereas realistic scattering times in the range of $\tau=50$ to $\tau=150$ fs [31] result in significant damping.

\section{APPENDIX G: COMPARISON OF $\mathrm{Cd}_{3} \mathrm{As}_{2}$ AND GRAPHENE IN THE SUPERCRITICAL REGIME}

In this section, we compare the HHG performance of a 250-nm-thick $\mathrm{Cd}_{3} \mathrm{As}_{2}$ thin film with a single graphene layer under similar driving conditions and doping. Figure 11 shows that despite the ability of monolayer graphene in producing prominent harmonics beyond the 31st order, both the energy spectral density (left vertical axis) and the energy conversion efficiency (right vertical axis) of the HHG from graphene is as much as 100 times smaller than that of $\mathrm{HHG}$ from the 250-nm-thick $\mathrm{Cd}_{3} \mathrm{As}_{2}$ thin film considered in this work. The results shown here indeed demonstrate that in the supercritical regime, the $\mathrm{HHG}$ spectra $3 \mathrm{D} \mathrm{DSM} \mathrm{Cd} \mathrm{As}_{2}$ is qualitatively similar to the HHG spectra of monolayer graphene with the added advantage of having a bulk interaction volume in contrast to a single atomic layer.

To further emphasize the advantage of HHG in 3D DSMs, we consider a hypothetical structure consisting of $N$ graphene layers sufficiently distant from each other such that the linear band structure still persists for each monolayer. Choosing this interlayer distance to be of the order of $10 \mathrm{~nm}$ and given that the driving wavelength is of the order of $100 \mu \mathrm{m}$, we assume 


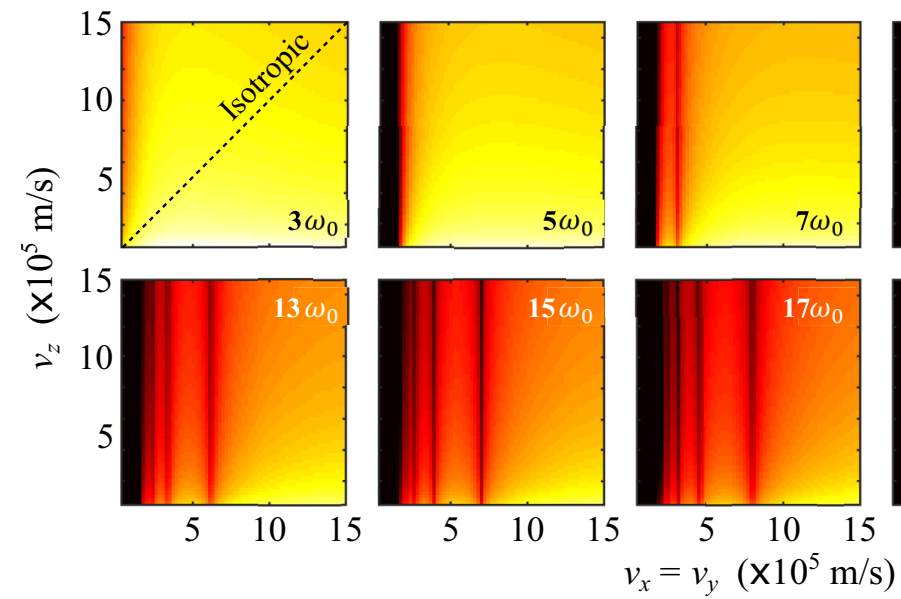

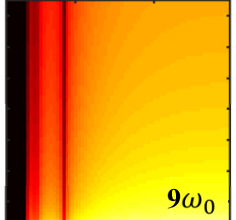

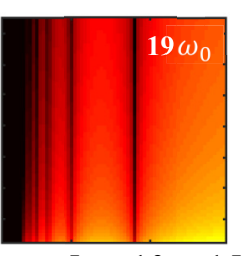

$5 \quad 10 \quad 15$

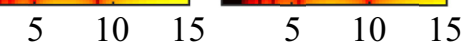

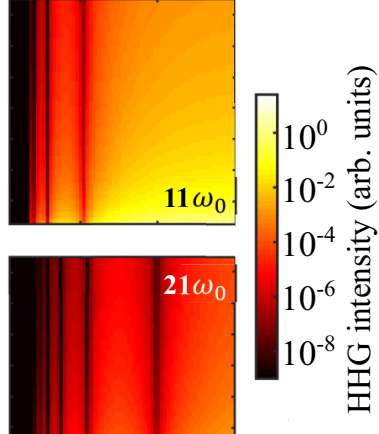

FIG. 12. 3D DSM HHG intensity in the supercritical regime as a function of the Fermi velocities in each direction, as computed using Eqs. (2) and (3). We assume $v_{x}=v_{y}$ for simplicity. We consider a Fermi energy $\mathcal{E}_{\mathrm{F}}=250 \mathrm{meV}$, a temperature $T=0 \mathrm{~K}$, and no carrier scattering. The incident field is a 2-ps-long, 1-THz-centered pulse linearly polarized along $x$ with a peak field strength $E_{x, 0}=10 \mathrm{MV} / \mathrm{m}$. The color maps indicate that the performance of HHG varies greatly depending on Fermi velocity values and anisotropy.

that all layers within the stack experience the same driving laser fields. Further making the assumption that the output HHG radiation does not interact with the rest of the stack and that the radiation emerging from each layer is superposed in a perfectly coherent manner, we find that the HHG intensity scales as $N^{2}$. Even under these idealistic hypothetical conditions, we find that a stack of at least $N=9$ layers of the graphene-spacer heterostructure is required to match the HHG intensities and energy conversion efficiencies produced by the 250-nm-thick $\mathrm{Cd}_{3} \mathrm{As}_{2}$ thin film.
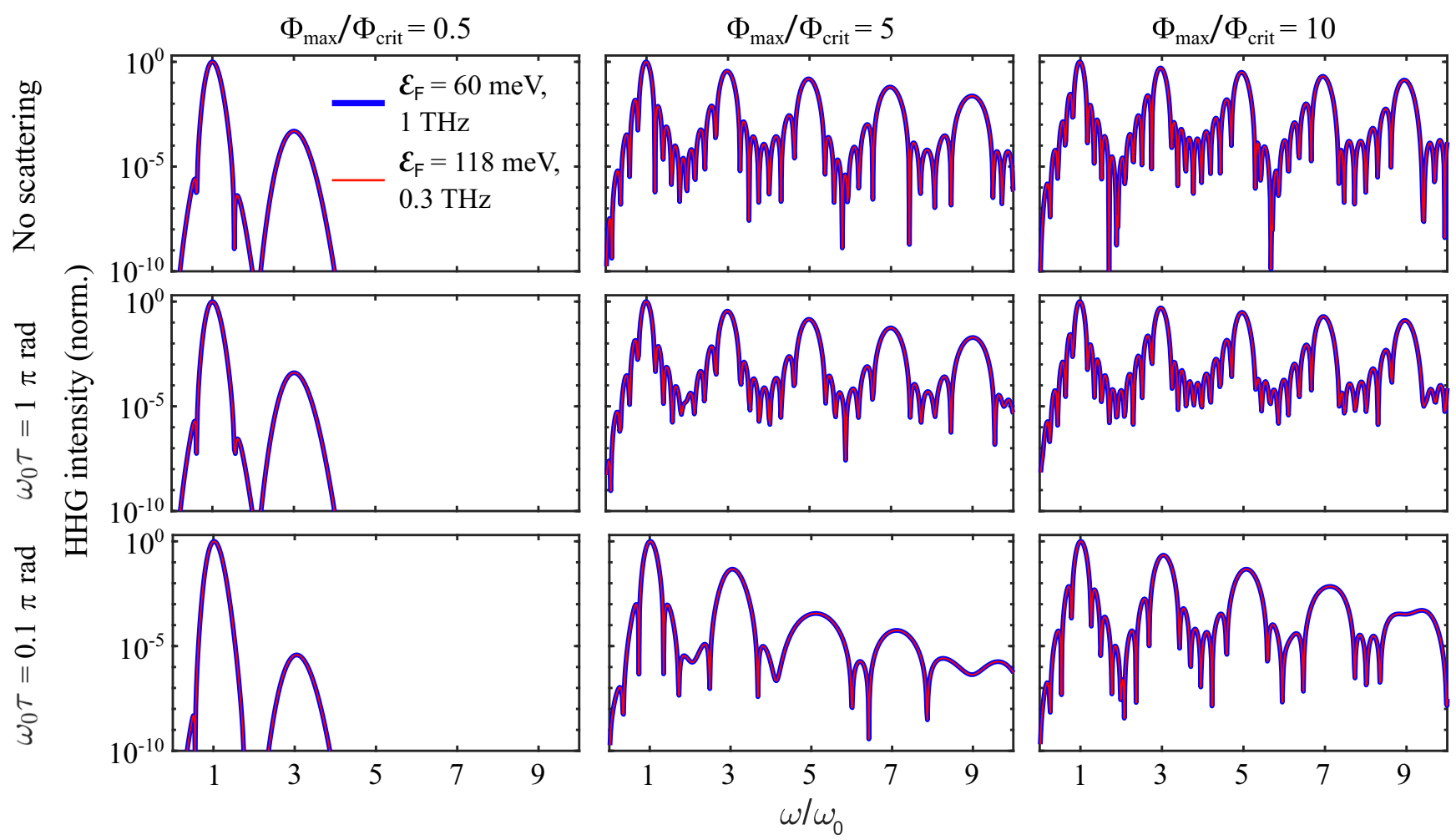

FIG. 13. Harmonic spectra for different combinations of parameters. For all panels, the spectrum represented by the thin red curve corresponds to the following parameters: $\mathcal{E}_{\mathrm{F}}=118 \mathrm{meV}, f_{0}=\omega_{0} / 2 \pi=0.3 \mathrm{THz}$, and $v_{x}=v_{y}=v_{z}=0.78 \times 10^{6} \mathrm{~m} / \mathrm{s}$. The spectrum denoted by the thick blue line corresponds to the following parameters: $\mathcal{E}_{\mathrm{F}}=60 \mathrm{meV}, f_{0}=\omega_{0} / 2 \pi=1 \mathrm{THz}$, and $\left(v_{x}, v_{y}, v_{z}\right)=(1.28,1.3,0.327) \times 10^{6}$ $\mathrm{m} / \mathrm{s}$. For both cases, a two-cycle-long (intensity FWHM) driving pulse is considered. In each panel, the scattering times $\tau$ and potential amplitudes $\Phi_{\max }$ are chosen such that the quanitites $\omega_{0} \tau$ and $\Phi_{\max } / \Phi_{\text {crit }}$ are the same for both sets of parameters. We see that the normalized harmonic spectra for both sets of parameters overlap exactly in all panels. All results shown here are computed at $T=0 \mathrm{~K}$ using our closed-form analytical expressions given by Eqs. (2) and (3). 


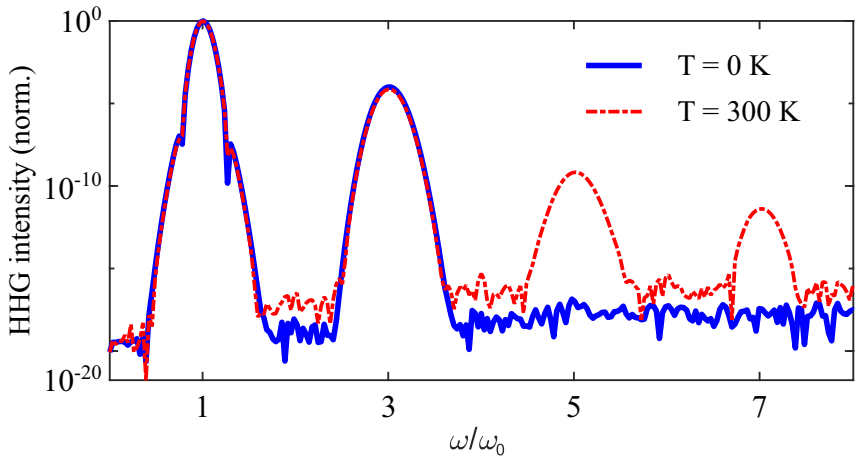

FIG. 14. Demonstration that the deviation of the predicted number of harmonics in Fig. 4 from experimentally reported results in Ref. [32] is due to temperature difference. No intraband harmonics above the third order are predicted in the low-temperature limit $T=0 \mathrm{~K}$ (solid blue curve). At the reported experimental temperature $T=300 \mathrm{~K}$, intraband-only simulations using the same set of parameters indicate that higher order intraband harmonics are generated (red dash-dotted curve) — consistent with the result reported in Ref. [32]. We consider the following parameters: $f_{0}=\omega_{0} / 2 \pi=$ $0.3 \mathrm{THz}, \mathcal{E}_{\mathrm{F}}=118 \mathrm{meV}, v_{x}=v_{y}=v_{z}=0.78 \times 10^{6} \mathrm{~m} / \mathrm{s}, E_{0, x}=6$ $\mathrm{MV} / \mathrm{m}$, and $\tau=10 \mathrm{fs}$. We use a 14.7-ps-long (intensity FWHM) driving $\mathrm{THz}$ pulse.

\section{APPENDIX H: EFFECT OF FERMI VELOCITY ANISOTROPY ON HHG INTENSITY}

Figure 12 shows that anisotropic Fermi velocities have a large effect on the efficiency of HHG for a fixed driving field strength. As the potential has the form $\Phi(t) \equiv \sqrt{\sum_{i} v_{i}^{2} A_{i}^{2}}$, a higher $v_{x}$ in this case (where the field is polarized along $x$ ) implies that a lower field strength is required to access the supercritical regime. Hence, a DSM with higher $v_{x}$ will operate deeper in the supercritical regime for an $x$-polarized field, which translates into more intense HHG and more prominent higher order harmonics in general. This also explains why higher order harmonics rapidly emerge at a low field strength for $\mathrm{Cd}_{3} \mathrm{As}_{2}$ as compared to $\mathrm{Na}_{3} \mathrm{Bi}$ and graphene, as our results in Figs. 3(a)-3(c) indicate (since $\mathrm{Cd}_{3} \mathrm{As}_{2}$ has the higher Fermi velocity among these materials in the direction of the driving laser polarization). Deep in the supercritical regime, we have shown that the induced current tends toward a square wave profile saturating at a finite amplitude $g e \mathcal{E}_{\mathrm{F}}^{3} /\left(6 \pi^{2} \hbar^{3} v_{y} v_{z}\right)$. We note that this saturation amplitude is larger for smaller values of $v_{z}$. This explains why higher intensity harmonics appear for larger $v_{x}, v_{y}$ values and small $v_{z}$ values in Fig. 12. The substantial influence on the Fermi velocity v over the nonlinear optical properties of 3D DSMs motivates the development of band-structure engineering methods as a means to enhance the HHG intensity and to alleviate the conditions necessary to operate deep within the supercritical regime.

\section{APPENDIX I: PARAMETERS USED TO PLOT FIG. 4}

The color map in Fig. 4 was plotted using our closedform analytical expressions given by Eqs. (2) and (3), corresponding to the low-temperature limit $T \rightarrow 0 \mathrm{~K}$ and when the intraband contribution to the current dominates, that is, $\hbar \omega_{0} \ll 2 \mathcal{E}_{\mathrm{F}}$. We fixed the central frequency of the driving field at $f_{0}=\omega_{0} / 2 \pi=1 \mathrm{THz}$, and considered a doping level $\mathcal{E}_{\mathrm{F}}=60 \mathrm{meV}$ and Fermi velocities $\left(v_{x}, v_{y}, v_{z}\right)=$ $(1.28,1.3,0.33) \times 10^{6} \mathrm{~m} / \mathrm{s}$, which correspond to the same parameters used in Fig. 1. The driving field is linearly polarized along $x$, and the pulse duration is 2 ps (intensity FWHM).

The data point that represents Fig. 1(b) in Fig. 4 is plotted using $E_{0, x}=10 \mathrm{MV} / \mathrm{m}$ and a realistic scattering time $\tau=150$ fs [31] [note that Fig. 1(b) assumed no scattering], which gives $\Phi_{\max } / \Phi_{\text {crit }} \approx 33.95$ and $\omega_{0} \tau=0.3 \pi \mathrm{rad}$. We used the following parameters to plot the data point that represents Ref. [31]: central frequency of the driving pulse $f_{0}=\omega_{0} / 2 \pi=0.8 \mathrm{THz}$, doping level $\mathcal{E}_{\mathrm{F}}=50 \mathrm{meV}$, isotropic Fermi velocities $v_{x}=v_{y}=v_{z}=0.93 \times 10^{6} \mathrm{~m} / \mathrm{s}$, a peak driving field amplitude $E_{0, x}=0.6 \mathrm{MV} / \mathrm{m}$ inside the material, and a scattering time $\tau=145$ fs. These values yield $\Phi_{\max } / \Phi_{\text {crit }} \approx$ 2.22 and $\omega_{0} \tau \approx 0.232 \pi \mathrm{rad}$. We used the following parameters to plot the data point that represents Ref. [32]: central frequency of the driving pulse $f_{0}=\omega_{0} / 2 \pi=0.3 \mathrm{THz}$, doping level $\mathcal{E}_{\mathrm{F}}=118 \mathrm{meV}$, isotropic Fermi velocities $v_{x}=$ $v_{y}=v_{z}=0.78 \times 10^{6} \mathrm{~m} / \mathrm{s}$, a peak driving field amplitude $E_{0, x}=6 \mathrm{MV} / \mathrm{m}$ inside the material, and a scattering time $\tau=10 \mathrm{fs}$. These values yield $\Phi_{\max } / \Phi_{\text {crit }} \approx 21.04$ and $\omega_{0} \tau=$ $0.006 \pi \mathrm{rad}$.

It should be noted that as long as two different combinations of parameters share the same values of $\Phi_{\max } / \Phi_{\text {crit }}, \omega_{0} \tau$, and are driven by the same number of laser field cycles, their normalized HHG spectra will be practically identical, as we show in Fig. 13. This justifies plotting markers representing Fig. 1(b) and Refs. [31] and [32] in Fig. 4, despite having different parameter combinations with respect to each other, as well as the parameters used to plot the color map.

In Fig. 4, only harmonics up to third order are predicted when the parameters from Ref. [32] were considered. However, up to the seventh-order harmonic was demonstrated experimentally. This deviation in the number of predicted harmonics from the reported result is due to our evaluation of Fig. 4 using the expressions given by Eqs. (2) and (3), which are only valid at $T=0 \mathrm{~K}$. However, the temperature in the experiment was $T=300 \mathrm{~K}$, and we show in Fig. 14 that this difference in temperature accounts for the observed deviation. While the HHG spectrum computed at $T=0 \mathrm{~K}$ (solid blue curves) indicates that no intraband harmonics above third order are generated for the parameters reported in Ref. [32], our intraband-only numerical simulations at $T=300 \mathrm{~K}$ (red dash-dotted curves), using Eqs. (A9a) and (A9b) with $\Gamma_{\mathbf{p}}(t)=$ 0 at all times (i.e., interband polarization switched off), show that higher order harmonics beyond the third order are indeed generated. This temperature difference should also explain the deviation of the number of predicted harmonics from the results reported in Ref. [31]. Additionally, we note that further deviations of our predictions from the experimental results reported in Ref. [31] could also arise due to the presence of substrates accompanying the $\mathrm{Cd}_{3} \mathrm{As}_{2}$ thin film. 
[1] D. N. Christodoulides, T. H. Coskun, M. Mitchell, and M. Segev, Theory of Incoherent Self-Focusing in Biased Photorefractive Media, Phys. Rev. Lett. 78, 646 (1997).

[2] A. Ramaniuk, P. S. Jung, D. N. Christodoulides, W. Krolikowski, and M. Trippenbach, Absorption-mediated stabilization of nonlinear propagation of vortex beams in nematic liquid crystals, Opt. Commun. 451, 338 (2019).

[3] B. Freedman, G. Bartal, M. Segev, R. Lifshitz, D. N. Christodoulides, and J. W. Fleischer, Wave and defect dynamics in nonlinear photonic quasicrystals, Nature (London) 440, 1166 (2006).

[4] D. N. Christodoulides, F. Lederer, and Y. Silberberg, Discretizing light behaviour in linear and nonlinear waveguide lattices, Nature (London) 424, 817 (2003).

[5] J. W. Fleischer, M. Segev, N. K. Efremidis, and D. N. Christodoulides, Observation of two-dimensional discrete solitons in optically induced nonlinear photonic lattices, Nature (London) 422, 147 (2003).

[6] B. Liu, B. Sain, B. Reineke, R. Zhao, C. Meier, L. Huang, Y. Jiang, and T. Zentgraf, Nonlinear wavefront control by geometric-phase dielectric metasurfaces: Influence of mode field and rotational symmetry, Adv. Opt. Mater. 8, 1902050 (2020).

[7] D. Kim, H. Chung, J. Yu, I. Hwang, S. Park, F. Demmerle, G. Boehm, M.C. Amann, M. A. Belkin, J.Y. Jung et al., Spincontrolled nonlinear harmonic generations from plasmonic metasurfaces coupled to intersubband transitions, Adv. Opt. Mater. 8, 2000004 (2020).

[8] E. Almeida, O. Bitton, and Y. Prior, Nonlinear metamaterials for holography, Nat. Commun. 7, 12533 (2016).

[9] N. Yoshikawa, T. Tamaya, and K. Tanaka, High-harmonic generation in graphene enhanced by elliptically polarized light excitation, Science 356, 736 (2017).

[10] J. D. Cox, A. Marini, and F. J. García de Abajo, Plasmonassisted high-harmonic generation in graphene, Nat. Commun. 8, 14380 (2017).

[11] H. A. Hafez, S. Kovalev, J.-C. Deinert, Z. Mics, B. Green, N. Awari, M. Chen, S. Germanskiy, U. Lehnert, J. Teichert et al., Extremely efficient terahertz high-harmonic generation in graphene by hot Dirac fermions, Nature (London) 561, 507 (2018).

[12] N. Rivera, L. J. Wong, M. Soljačić, and I. Kaminer, Ultrafast Multiharmonic Plasmon Generation by Optically Dressed Electrons, Phys. Rev. Lett. 122, 053901 (2019).

[13] J. Itatani, J. Levesque, D. Zeidler, H. Niikura, H. Pépin, J. C. Kieffer, P. B. Corkum, and D. M. Villeneuve, Tomographic imaging of molecular orbitals, Nature (London) 432, 867 (2004).

[14] H. J. Wörner, J. B. Bertrand, D. V. Kartashov, P. B. Corkum, and D. M. Villeneuve, Following a chemical reaction using high-harmonic interferometry, Nature (London) 466, 604 (2010).

[15] E. Goulielmakis, Z.-H. Loh, A. Wirth, R. Santra, N. Rohringer, V. S. Yakovlev, S. Zherebtsov, T. Pfeifer, A. M. Azzeer, M. F. Kling et al., Real-time observation of valence electron motion, Nature (London) 466, 739 (2010).

[16] F. Calegari, D. Ayuso, A. Trabattoni, L. Belshaw, S. De Camillis, S. Anumula, F. Frassetto, L. Poletto, A. Palacios, P. Decleva et al., Ultrafast electron dynamics in phenylalanine initiated by attosecond pulses, Science 346, 336 (2014).
[17] S. Ghimire, A. D. DiChiara, E. Sistrunk, P. Agostini, L. F. Dimauro, and D. A. Reis, Observation of high-order harmonic generation in a bulk crystal, Nat. Phys. 7, 138 (2011).

[18] O. Schubert, M. Hohenleutner, F. Langer, B. Urbanek, C. Lange, U. Huttner, D. Golde, T. Meier, M. Kira, S. W. Koch et al., Sub-cycle control of terahertz high-harmonic generation by dynamical Bloch oscillations, Nat. Photon. 8, 119 (2014).

[19] T. T. Luu, M. Garg, S. Y. Kruchinin, A. Moulet, M. T. Hassan, and E. Goulielmakis, Extreme ultraviolet high-harmonic spectroscopy of solids, Nature (London) 521, 498 (2015).

[20] G. Ndabashimiye, S. Ghimire, M. Wu, D. A. Browne, K. J. Schafer, M. B. Gaarde, and D. A. Reis, Solid-state harmonics beyond the atomic limit, Nature (London) 534, 520 (2016).

[21] Y. S. You, D. A. Reis, and S. Ghimire, Anisotropic highharmonic generation in bulk crystals, Nat. Phys. 13, 345 (2017).

[22] Y. S. You, Y. Yin, Y. Wu, A. Chew, X. Ren, F. Zhuang, S. Gholam-Mirzaei, M. Chini, Z. Chang, and S. Ghimire, Highharmonic generation in amorphous solids, Nat. Commun. 8, 724 (2017).

[23] H. Liu, Y. Li, Y. S. You, S. Ghimire, T. F. Heinz, and D. A. Reis, High-harmonic generation from an atomically thin semiconductor, Nat. Phys. 13, 262 (2017).

[24] A. J. Uzan, G. Orenstein, Á. Jiménez-Galán, C. McDonald, R. E. F. Silva, B. D. Bruner, N. D. Klimkin, V. Blanchet, T. Arusi-Parpar, M. Krüger et al., Attosecond spectral singularities in solid-state high-harmonic generation, Nat. Photon. 14, 183 (2020).

[25] Y. W. Kim, T.-J. Shao, H. Kim, S. Han, S. Kim, M. Ciappina, X.-B Bian, and S.-W. Kim, Spectral interference in high harmonic generation from solids, ACS Photon. 6, 851 (2019).

[26] S. Han, L. Ortmann, H. Kim, Y. W. Kim, T. Oka, A. Chacon, B. Doran, M. Ciappina, M. Lewenstein, S.-W. Kim et al., Extraction of higher-order nonlinear electronic response in solids using high harmonic generation, Nat. Commun. 10, 3272 (2019).

[27] A. Chacón, D. Kim, W. Zhu, S. P. Kelly, A. Dauphin, E. Pisanty, A. S. Maxwell, A. Picón, M. F. Ciappina, D. E. Kim et al., Circular dichroism in higher-order harmonic generation: Heralding topological phases and transitions in Chern insulators, Phys. Rev. B 102, 134115 (2020).

[28] E. N. Osika, A. Chacón, L. Ortmann, N. Suárez, J. A. Pérez-Hernández, B. Szafran, M. F. Ciappina, F. Sols, A. S. Landsman, and M. Lewenstein, Wannier-Bloch Approach to Localization in High-Harmonics Generation in Solids, Phys. Rev. X 7, 021017 (2017).

[29] S. Ghimire and D. A. Reis, High-harmonic generation from solids, Nat. Phys. 15, 10 (2019).

[30] N. Tancogne-Dejean and A. Rubio, Atomic-like high-harmonic generation from two-dimensional materials, Sci. Adv. 4, eaao5207 (2018).

[31] B. Cheng, N. Kanda, T. N. Ikeda, T. Matsuda, P. Xia, T. Schumann, S. Stemmer, J. Itatani, N. P. Armitage, and R. Matsunaga, Efficient Terahertz Harmonic Generation with Coherent Acceleration of Electrons in the Dirac Semimetal $\mathrm{Cd}_{3} \mathrm{As}_{2}$, Phys. Rev. Lett. 124, 117402 (2020).

[32] S. Kovalev, R. M. A. Dantas, S. Germanskiy, J.-C. Deinert, B. Green, I. Ilyakov, N. Awari, M. Chen, M. Bawatna, J. Ling et al., Non-perturbative terahertz high-harmonic generation in 
the three-dimensional Dirac semimetal $\mathrm{Cd}_{3} \mathrm{As}_{2}$, Nat. Commun. 11, 2451 (2020).

[33] A. R. Wright, X. G. Xu, J. C. Cao, and C. Zhang, Strong nonlinear optical response of graphene in the terahertz regime, Appl. Phys. Lett. 95, 072101 (2009).

[34] S. Shareef, Y. S. Ang, and C. Zhang, Room-temperature strong terahertz photon mixing in graphene, J. Opt. Soc. Am. B 29, 274 (2012)

[35] F. Giorgianni, E. Chiadroni, A. Rovere, M. Cestelli-Guidi, A. Perucchi, M. Bellaveglia, M. Castellano, D. Di Giovenale, G. Di Pirro, M. Ferrario et al., Dirac surface states in $\mathrm{Bi}_{2} \mathrm{Se}_{3}$ topological insulator, Nat. Commun. 7, 11421 (2016).

[36] C. Wolff, C. Tserkezis, and N. A. Mortensen, Enhanced ponderomotive force in graphene due to interband resonance, New J. Phys. 21, 073046 (2019).

[37] P. A. D. Goncalves, N. Stenger, J. D. Cox, N. A. Mortensen, and S. Xiao, Strong light-matter interactions enabled by polaritons in atomically thin materials, Adv. Opt. Mater. 8, 1901473 (2020).

[38] G. Soavi, G. Wang, H. Rostami, A. Tomadin, O. Balci, I. Paradisanos, E. A. A. Pogna, G. Cerullo, E. Lidorikis, M. Polini et al., Hot electrons modulation of third-harmonic generation in graphene, ACS Photon. 6, 2841 (2019).

[39] G. Soavi, S. Dal Conte, C. Manzoni, D. Viola, A. Narita, Y. Hu, X. Feng, U. Hohenester, E. Molinari, D. Prezzi et al., Exciton-exciton annihilation and biexciton stimulated emission in graphene nanoribbons, Nat. Commun. 7, 11010 (2016).

[40] G. Soavi, G. Wang, H. Rostami, D. G. Purdie, D. De Fazio, T. Ma, B. Luo, J. Wang, A. K. Ott, D. Yoon et al., Broadband, electrically tunable third-harmonic generation in graphene, Nat. Nanotechnol. 13, 583 (2018).

[41] K. L. Ishikawa, Nonlinear optical response of graphene in time domain, Phys. Rev. B 82, 201402(R) (2010).

[42] K. L. Ishikawa, Electronic response of graphene to an ultrashort intense terahertz radiation pulse, New J. Phys. 15, 055021 (2013).

[43] L. A. Chizhova, F. Libisch, and J. Burgdörfer, High-harmonic generation in graphene: Interband response and the harmonic cutoff, Phys. Rev. B 95, 085436 (2017).

[44] A. Marini, J. D. Cox, and F. J. García de Abajo, Theory of graphene saturable absorption, Phys. Rev. B 95, 125408 (2017).

[45] O. V. Kotov and Y. E. Lozovik, Dielectric response and novel electromagnetic modes in three-dimensional Dirac semimetal films, Phys. Rev. B 93, 235417 (2016).

[46] K. J. A. Ooi, Y. S. Ang, Q. Zhai, D. T. H. Tan, L. K. Ang, and C. K. Ong, Nonlinear plasmonics of three-dimensional Dirac semimetals, APL Photon. 4, 034402 (2019).

[47] D. Neubauer, J. P. Carbotte, A. A. Nateprov, A. Löhle, M. Dressel, and A. V. Pronin, Interband optical conductivity of the [001]-oriented Dirac semimetal $\mathrm{Cd}_{3} \mathrm{As}_{2}$, Phys. Rev. B 93, 121202(R) (2016).

[48] X. Yuan, P. Cheng, L. Zhang, C. Zhang, J. Wang, Y. Liu, Q. Sun, P. Zhou, D. W. Zhang, Z. Hu et al., Direct observation of Landau level resonance and mass generation in Dirac semimetal $\mathrm{Cd}_{3} \mathrm{As}_{2}$ thin films, Nano Lett. 17, 2211 (2017).

[49] S. Nishihaya, M. Uchida, Y. Nakazawa, R. Kurihara, K. Akiba, M. Kriener, A. Miyake, Y. Taguchi, M. Tokunaga, and M.
Kawasaki, Quantized surface transport in topological Dirac semimetal films, Nat. Commun. 10, 2564 (2019).

[50] S. Nishihaya, M. Uchida, Y. Nakazawa, M. Kriener, Y. Kozuka, Y. Taguchi, and M. Kawasaki, Gate-tuned quantum hall states in Dirac semimetal $\left(\mathrm{Cd}_{1-x} \mathrm{Zn}_{x}\right)_{3} \mathrm{As}_{2}$, Sci. Adv. 4, eaar5668 (2018).

[51] T. Liang, J. Lin, Q. Gibson, T. Gao, M. Hirschberger, M. Liu, R. J. Cava, and N. P. Ong, Anomalous Nernst Effect in the Dirac Semimetal $\mathrm{Cd}_{3} \mathrm{As}_{2}$, Phys. Rev. Lett. 118, 136601 (2017).

[52] H. Hirori, A. Doi, F. Blanchard, and K. Tanaka, Single-cycle terahertz pulses with amplitudes exceeding $1 \mathrm{MV} / \mathrm{cm}$ generated by optical rectification in $\mathrm{LiNbO}_{3}$, Appl. Phys. Lett. 98, 091106 (2011).

[53] C. P. Hauri, C. Ruchert, C. Vicario, and F. Ardana, Strong-field single-cycle $\mathrm{THz}$ pulses generated in an organic crystal, Appl. Phys. Lett. 99, 161116 (2011).

[54] S. W. Huang, E. Granados, W. R. Huang, K.-H. Hong, L. E. Zapata, and F. X. Kärtner, High conversion efficiency, high energy terahertz pulses by optical rectification in cryogenically cooled lithium niobate, Opt. Lett. 38, 796 (2013).

[55] J. Fulop, L. Palfalvi, M. C. Hoffmann, and J. Hebling, Towards generation of mJ-level ultrashort $\mathrm{THz}$ pulses by optical recification, Opt. Express 19, 15090 (2011).

[56] J. Fulop, L. Palfalvi, G. Almasi, and J. Hebling, Design of high-energy terahertz sources based on optical rectification, Opt. Express 18, 12311 (2010).

[57] Z. K. Liu, J. Jiang, B. Zhou, Z. J. Wang, Y. Zhang, H. M. Weng, D. Prabhakaran, S.-K. Mo, H. Peng, P. Dudin et al., A stable three-dimensional topological Dirac semimetal $\mathrm{Cd}_{3} \mathrm{As}_{2}$, Nat. Mater. 13, 677 (2014).

[58] Z. K. Liu, B. Zhou, Y. Zhang, Z. J. Wang, H. M. Weng, D. Prabhakaran, S.-K. Mo, Z. X. Shen, Z. Fang, X. Dai et al., Discovery of a three-dimensional topological Dirac semimetal, $\mathrm{Na}_{3} \mathrm{Bi}$, Science 343, 864 (2014).

[59] E. Hendry, P. J. Hale, J. Moger, A. K. Savchenko, and S. A Mikhailov, Coherent Nonlinear Optical Response of Graphene, Phys. Rev. Lett. 105, 097401 (2010).

[60] J. E. Sipe and E. Ghahramani, Nonlinear optical response of semiconductors in the independent-particle approximation, Phys. Rev. B 48, 11705 (1993).

[61] K. Ahnert and M. Mulansky, Odeint-solving ordinary differential equations in $\mathrm{C}++$, in Numerical Analysis and Applied Mathematics ICNAAM 2011: International Conference on Numerical Analysis and Applied Mathematics, edited by T. E. Simos, G. Psihoyios, Ch. Tsitouras, and Z. Anastassi, AIP Conf. Proc. No. 1389 (AIP, New York, 2011), p. 1586.

[62] S. Feng and H. G. Winful, Spatiotemporal structure of isodiffracting ultrashort electromagnetic pulses, Phys. Rev. E 61, 862 (2000).

[63] G. Mur, Absorbing boundary conditions for the finite difference approximation of the time-domain electromagnetic-field equations, IEEE Trans. Electromagn. Compat. EMC-23, 377 (1981).

[64] R. Luebbers, F. P. Hunsberger, K. S. Kunz, R. B. Standler, and M. A. Schneider, Frequency-dependent finite-difference time-domain formulation for dispersive materials, IEEE Trans. Electromagn. Compat. 32, 222 (1990).

[65] V. Nayyeri, M. Soleimani, and O. M. Ramahi, Modeling graphene in the finite-difference time-domain method using a 
surface boundary condition. IEEE Trans. Antennas Propag. 61, 4176 (2013).

[66] S. G. Johnson, Cubature integration, http://ab-initio.mit.edu/ wiki/index.php/Cubature_(Multi-dimensional_integration).

[67] M. Baudisch, A. Marini, J. D. Cox, T. Zhu, F. Silva, S. Teichmann, M. Massicotte, F. Koppens, L. S. Levitov, F. J.
García de Abajo et al., Ultrafast nonlinear optical response of Dirac fermions in graphene, Nat. Commun. 9, 1018 (2018).

[68] G. S. Jenkins, C. Lane, B. Barbiellini, A. B. Sushkov, R. L. Carey, F. Liu, J. W. Krizan, S. K. Kushwaha, Q. Gibson, T.-R. Chang et al., Three-dimensional Dirac cone carrier dynamics in $\mathrm{Na}_{3} \mathrm{Bi}$ and $\mathrm{Cd}_{3} \mathrm{As}_{2}$, Phys. Rev. B 94, 085121 (2016). 